\title{
On a laser $(3+1)$-dimensional vectorial cubic-quintic complex Ginzburg-Landau equation and modulational instability
}

\author{
Alain Djazet ${ }^{1,2 *}$, Serge I. Fewo ${ }^{1 \dagger}$, Conrad B. Tabi ${ }^{3 \ddagger}$ and Timoléon C. Kofané $e^{1,2,3 \S}$ \\ ${ }^{1}$ Laboratoire de Mécanique, Département de Physique, Faculté des Sciences, Université de Yaoundé I, \\ B.P. 812 Yaoundé, Cameroun \\ ${ }^{2}$ Centre d'Excellence Africain en Technologies de l'Information et de la Communication (CETIC), \\ University of Yaounde I, Cameroon \\ ${ }^{3}$ Botswana International University of Science and Technology, Private Bag 16 Palapye, Botswana
} September 11, 2019

\section{Abstract}

Interaction of an electromagnetic field with matter in a laser cavity without the assumption

*nguitewo@gmail.com (A. Djazet)

$\dagger^{\dagger}$ sergefewo@yahoo.fr (S. I. Fewo)

${ }^{\ddagger}$ conrad@aims.ac.za or tabic@biust.ac.bw (C. B. Tabi)

§tckofane@yahoo.com (T. C. Kofané) 
Keywords: Laser cavity; Two-level Maxwell-Bloch equations; Complex Ginzburg-Landau equation; Modulational instability; Pattern formation.

\section{Introduction}

New theoretical approaches, experimental analyses, and systematic use of computer science in data processing have been developed during the past 20 years in several types of lasers, which are very complex devices, having a rich temporal, spatial, and spatiotemporal dynamics [1]. These different types of lasers can be classified into [2] Class A (for example, dye lasers) [3, 4], Class B (semiconductor lasers, $\mathrm{CO}_{2}$ lasers, and solid-sate lasers) [5, 6], and Class $\mathrm{C}$ (the only example is the far-infrared lasers) [7], depending on the decay rate of the photons, the carriers, and the material polarization. However, this classification is not applicable to inhomogeneously broadened lasers that included He-Ne, argon-ion, and Xe lasers, for example. Comparing these lasers, different dynamical features have been described, including instabilities, cascades of bifurcations, multistability, and sudden chaotic transitions [1]. Many other fascinating features and properties concerned with chaotic dynamics have been extensively addressed in relevant semiconductor laser systems, because of their potential applications in chaotic optical communications [8]. Further studies have suggested that optical cavities, also called cavity solitons, are present in a large variety of externally driven optical systems. However, their existence in laser systems is limited to the well-known laser with saturable absorbers, two-photon lasers, lasers with dense amplifying medium, or lasers pumped by squeezed vacuum [9].

Several models have been proposed to describe how the spatiotemporal dynamics emerges in large-aperture lasers. For example, the two-photon lasers have been the subject of continued theoretical attention since the early days of the laser era. The theoretical interest of the twophoton laser lies in the intrinsic nonlinear nature of the two-photon interaction. The most successful theoretical approach is given by the Maxwell-Bloch (MB) equations. In fact, the laser is a system where the number of photons is much larger than one, thus allowing a semi-classical treatment of the electromagnetic field inside the cavity through the Maxwell equations, which has been developed by Lamb [10] and independently by Haken [11]. The semi-classical laser 
theory ignores the quantum-mechanical nature of the electromagnetic field, and the amplifying medium is modeled quantum mechanically, as a collection of two-level atoms through the Bloch equations.

The linear analysis and numerical integration of the full MB equations [12] have been used to interpret the features of the experiment that cannot be fully understood with a perturbative model, such as the observed evolution from order to fully developed turbulence as the Fresnel number increases up to a critical control-parameter threshold [13]. In addition, it has been shown that the MB equations with homogeneous line broadening are appropriate for the description of the amplification of short pulses in the multilevel atomic iodine amplifier [14]. Some prototype of nonlinear evolution equations has been constructed by singular perturbation methods, using the MB equations as the starting point, in order to reproduce the spatiotemporal dynamics of the large-aperture lasers.

The first class of prototype equations which describe, for example, the class-A laser pattern dynamics, such as the multi-transverse-mode lasers, is the cubic complex Ginzburg-Landau (CGL) equation. In fact, the existence of a vortex solution of the laser equations, the stability of symmetric vortex lattices in the laser beams, the transition to nonsymmetric patterns dominated by titled waves, and to disordered spatial distribution have been well-reproduced by the cubic CGL equation $[15,16]$. To prevent the "blowup" of the solutions of the cubic CGL equation for negative detuning, the laser cubic CGL equation, which possesses fourth- and higher-order diffusion terms and which describes correctly the excitation of transverse modes and structure formation in a laser, has been derived [17]. It should also be mentioned that the adiabatic elimination of irrelevant variables has been shown to be very sensitive to the method used for the perturbation expansions in the case of partial differential equations which describe laser dynamics. That is why the center manifold theorem for the elimination of irrelevant variables has been used, leading to the cubic CGL equation in the small-field limit. The particular feature of the center manifold theory is that it is a solid mathematical framework within which the fast variables as well as the characteristic scaling of the long-term dynamics are properly determined [18]. It has also been shown that the cubic-quintic CGL equation is a continuous approximation to the dynamics of the field in a passively mode-locked laser [19]. 
The second class of prototype equations which provides the generic description of transverse pattern formation in wide aperture, single longitudinal mode, two-level lasers, when the laser is operating near peak gain, is the complex Swift - Hohenberg equation for class A and C lasers [20]. Indeed, the complex Swift - Hohenberg equation comes naturally as a solvability condition for the existence of solutions to the MB laser equations in the form of asymptotic series in powers of the small detuning parameter [20]. In addition, when the laser pattern dynamics is sensitive to the degree of stiffness of the original physical problem, such as in the class-B lasers, the amplitude equations are the complex Swift - Hohenberg equation coupled to a mean flow [20], which is consistent with the observation that the population inversion variable in the MB laser equations acts as a weakly damped mode. Otherwise, the Swift-Hohenberg equation has been considered for a passive optical cavity driven by an external coherent field, valid close to the onset of optical bistability [21]. Moreover, theoretical studies of spatiotemporal structures of lasers with a large Fresnel number of the laser cavity have been successfully described in the cases in which two coupled fields are involved in the dynamics for class-B lasers. For example, it has been shown that the homogeneous steady-state solution may be destabilized by two generic instabilities. The first is a long wavelength instability which is related to the phase invariance of the electromagnetic field and is described by a scalar field obeying the Kuramoto-Shivasinsky equation. The second is a short wavelength instability which corresponds to a Hopf bifurcation and is described by a complex field which obeys a Swift-Hohenberg equation.

The third class of prototype equations which contains a phenomenological aspect and whose use in the theoretical description of the pulse dynamics in a mode-locked laser was pioneered by Haus and Mecozzi [22]. Assuming that only one polarization state plays a role and that the change of the pulse per round trip is small, so that one can replace the discrete laser components with continuous approximations, Haus and Mecozzi [22] obtained a master equation which is nothing but the stationary version of the cubic CGL equation. The coefficients that appear in the model were related to the physical parameters in a rather phenomenological way [22, 23].

All these three classes of prototype equations are scalar since it is usually considered that the polarization degree of freedom of the electromagnetic field is fixed either by material anisotropies or by experimental arrangement. Thus, the description of the dynamics is done in terms of a 
scalar field. It has been shown that the cavity-synchronous phase or amplitude modulation technique transforms passively mode-locked optical oscillators into actively mode-locked lasers [24]. Mixing passive and active mode-locking in the same device results in a new class of optical oscillators capable of generating short pulses. To model this laser system, as an example, the scalar cubic-quintic CGL equation has been used with terms corresponding to active mode-locking in addition to the usual passive mode-locking terms [25]. However, the inclusion of a quintic saturating term in the scalar cubic-quintic CGL equation was shown to be essential for the stability of pulsed solutions [26]. Since the scalar cubic-quintic CGL equation is non-integrable, which means that general analytical solutions are not available, selected analytical solutions can only be found for specific relations between the equation parameters. More complicated solutions for the cubic-quintic CGL equations, such as pulsating, creeping, or exploding solutions have been reported numerically [27]. It is also well known that laser systems are made of several components, an accurate model then should involve consecutive sets of propagation equations. Models can be vectorial, when the polarization nature of light is involved, and can also include the delayed response of the saturable absorber and gain medium. The possibility of vectorial topological defects which are not predictable by the scalar theory were first analyzed by Gil [28]. Using standard perturbative nonlinear analysis performed near the laser threshold, Gil derived a $(3+1)$-dimensional $((3+1) \mathrm{D})$ vectorial cubic CGL equation when considering the interaction of an electromagnetic field with matter in a laser cavity without the assumption of a fixed direction of the transverse electric field. Different kinds of pattern formation are present in the dynamic states of the one-spatial dimension (localized structures) [29] and of the two-spatial dimensions (topological defects) [30, 31, 32, 33] for the vectorial cubic CGL equation. Examples are the synchronization properties of spatiotemporally chaotic states [30], the identification of a transition from a glass to a gas phase [31], and the formation and annihilation processes leading to the different types of defects [32]. In addition, creation and annihilation processes of different kinds of vector defects, as well as a transition between different regimes of spatiotemporal dynamics have been described [33].

The objective of the present work is to get a qualitative understanding of the physical processes involved in spatial pattern formation from a two-level atomic system controlled by 
an intense laser field. The approach taken here parallels that of Gil [28] for the vectorial cubic CGL equation. We start with the Maxwell-Bloch equations describing the propagation of a slowly varying field envelope through a collection of two-level atoms when the interaction of an electromagnetic field with matter in a laser cavity is considered without the assumption of a fixed direction of the transverse electric field. Then, we report on the derivation of the laser $(3+1)$ D vectorial cubic-quintic CGL equation. Furthermore, we discuss, theoretically and numerically, modulational instability (MI) of plane waves on this equation. MI, which is an indispensable mechanism for understanding pattern formation in a uniform medium, is a process in which the amplitude and phase modulations of a wave grow under the combined effects of nonlinearity and diffraction or dispersion in a spatially nonlinear field [34, 35, 36, 37, 38, 39]. We examine their stability by means of both the rigorous analysis of linearized equations for small perturbations, and in direct numerical simulations to support our analytical results.

The rest of the paper is organized as follows. In Sec. II, we derive the laser $(3+1)$ vectorial cubic-quintic CGL equation which describes the laser pattern dynamics. In Sec. III, the linear stability analysis of MI is performed, and the instability zones, as well as the analytical expressions of the gain of MI are obtained. In Sec. IV, we focus on the role played by the loss/gain coefficient. Then, in Sec. V, we perform direct numerical integrations to check the validity of the MI conditions found analytically. Section VI concludes the paper.

\section{Derivation of the laser $(3+1) \mathrm{D}$ vectorial cubic-quintic CGL equation}

We consider the behavior of a slowly varying field envelope through a collection of two-level atoms with a transition frequency $w_{a}$ between the lasing levels, and relaxation rate $\gamma_{\perp}$ and $\gamma_{\|}$ for the polarization and the population inversion, respectively, and when the interaction of an electromagnetic field with matter in a laser cavity is considered without the assumption of a fixed direction of the transverse electric field. The basic equations of motion are the well-known MB equations [28, 40] written as

$$
\frac{\partial^{2} \mathbf{E}}{\partial t^{2}}=-\mu_{0} c^{2} \frac{\partial^{2} \mathbf{P}}{\partial t^{2}}+c^{2}\left[\nabla^{2} \mathbf{E}-\nabla(\nabla \cdot \mathbf{E})\right]-\kappa \frac{\partial \mathbf{E}}{\partial t},
$$




$$
\frac{\partial^{2} \mathbf{P}}{\partial t^{2}}=-\gamma_{\perp} \frac{\partial \mathbf{P}}{\partial t}-w_{a}^{2} \mathbf{P}-g D \mathbf{E}
$$

$$
\frac{\partial D}{\partial t}=-\gamma_{\|}\left(D-D_{0}\right)+\frac{2}{\hbar w}\left(\mathbf{E} \cdot \frac{\partial \mathbf{P}}{\partial t}\right),
$$

157

169

$$
\mathbf{P}_{2}^{1}=\frac{i g}{\gamma_{\perp} w_{a}}\left(D_{1}^{0} \mathbf{E}_{1}^{1}\right)
$$

170

$$
\mathbf{P}_{3}^{1}=\frac{i g}{\gamma_{\perp} w_{a}}\left(D_{2}^{0} \mathbf{E}_{1}^{1}+D_{2}^{2} \mathbf{E}_{1}^{-1}\right)
$$

171

$$
\mathbf{P}_{3}^{3}=\frac{i g}{\left(8 w_{a}-3 i \gamma_{\perp}\right)}\left(D_{2}^{2} \mathbf{E}_{1}^{1}\right)
$$


172 and

$$
D_{1}^{0}=D_{0}, \quad D_{2}^{0}=\frac{2 i}{\hbar \gamma_{\|}}\left(\mathbf{P}_{1}^{1} \mathbf{E}_{1}^{-1}-\mathbf{P}_{1}^{-1} \mathbf{E}_{1}^{1}\right), \quad D_{2}^{2}=\frac{2 i}{\hbar\left(\gamma_{\|}+2 i w_{a}\right)}\left(\mathbf{P}_{1}^{1} \mathbf{E}_{1}^{1}\right)
$$

173 (see Appendix).

174

and

$$
Z=\epsilon^{2} z, \quad T=\epsilon^{2} t .
$$

with

$$
\left(\begin{array}{l}
\mathbf{E}_{1} \\
\partial_{t} \mathbf{E}_{1} \\
\mathbf{P}_{1} \\
\partial_{t} \mathbf{P}_{1} \\
D_{1}
\end{array}\right)=\left(\begin{array}{l}
\mathbf{A} \\
i w_{a} \mathbf{A} \\
\frac{1}{\mu_{0} c^{2}}\left(-1+\frac{i k}{w_{a}}\right) \mathbf{A} \\
\frac{i w_{a}}{\mu_{0} c^{2}}\left(-1+\frac{i k}{w_{a}}\right) \mathbf{A} \\
0
\end{array}\right) \mathrm{e}^{i\left(w t-k_{c} z\right)}+\text { c.c. }
$$


with $\mathbf{A} \perp \mathbf{Z}$, where $\mathbf{A}$ is slowly varying field amplitude in space and time. After inserting Eqs. (6)-(8) into the MB equations, rearranging terms and making use of Eqs. (3), (5) and (9), and identifying the coefficients of powers $\epsilon$ at each order, we obtain, by applying the solvability conditions at $0\left(\epsilon^{2}\right)$ and $0\left(\epsilon^{3}\right)$, the laser $(3+1) D$ vectorial cubic-quintic CGL equation

$$
\begin{aligned}
\frac{\partial \mathbf{A}}{\partial T} & =z_{1} \mathbf{A}-z_{2}\left(\frac{\partial}{\partial \mathbf{Z}}+\frac{i}{2 k_{c}} \nabla_{\perp}^{2}\right) \mathbf{A}+z_{3}\left(\frac{\partial}{\partial Z}+\frac{i}{2 k_{c}} \nabla_{\perp}^{2}\right)^{2} \mathbf{A}-z_{4}\left(\mathbf{A} \cdot \mathbf{A}^{*}\right) \mathbf{A}-z_{5}(\mathbf{A} \cdot \mathbf{A}) \mathbf{A}^{*} \\
& +z_{6}\left(\mathbf{A}^{2} \cdot \mathbf{A}^{* 2}\right) \mathbf{A}+z_{7}\left(\mathbf{A}^{3} \cdot \mathbf{A}^{*}\right) \mathbf{A}^{*},
\end{aligned}
$$

where $\nabla_{\perp}^{2}=\frac{\partial^{2}}{\partial X^{2}}+\frac{\partial^{2}}{\partial Y^{2}}$, represents a two-dimensional Laplacian operator and the asterisk $(*)$ stands for the complex conjugate, while the coefficients are given in the Appendix. Eq. (10) describes the behavior of the electric field in the medium (dielectric medium). When coefficients $z_{6}=z_{7}=0$, in Eq. (10), we recover the laser $(3+1) D$ vectorial cubic CGL equation that was introduced early by Gil [28] as a vector order parameter for an unpolarized laser and its vectorial topological defects.

Due to the highly nonlinear nature of Eq. (10), we introduce a number of useful simplifications: (i) we use the traditional uniform field limit which requires that both the mirror transmittivity and the gain per pass of the active medium be small, while their ratio may be arbitrary but finite; (ii) a large free spectral range; (iii) the number of modes that are significantly excited is manageably small [41]; (iv) the fourth-order derivative has been neglected [28]. In this way, the new field amplitude obeys the equation of motion

$$
\mathbf{A}=\mathbf{B}(X, Y, T) \exp (-i \Delta Z)
$$

where the amplitude $B(X, Y, T)$ is governed by the equation

$$
\frac{\partial \mathbf{B}}{\partial T}=c_{1} \mathbf{B}+c_{2} \nabla^{2} \mathbf{B}-c_{3}\left(\mathbf{B} \cdot \mathbf{B}^{*}\right) \mathbf{B}-c_{4}(\mathbf{B} \cdot \mathbf{B}) \mathbf{B}^{*}+c_{5}\left(\mathbf{B}^{2} \cdot \mathbf{B}^{* 2}\right) \mathbf{B}+c_{6}\left(\mathbf{B}^{3} \cdot \mathbf{B}^{*}\right) \mathbf{B}^{*}
$$

where $c_{1}=z_{1}+\Delta\left(-\Delta z_{3}+i z_{2}\right), \quad c_{2}=\frac{\left(2 \Delta z_{3}-i z_{2}\right)}{2 k_{c}}, \quad c_{3}=z_{4} ; \quad c_{4}=z_{5}, \quad c_{5}=z_{6}, \quad c_{6}=z_{7}$. with $\mathbf{B} \perp \mathbf{Z}$. Considering the case where $\mathbf{B}$ has two complex components such as $\mathbf{B}=\left(B_{x}, B_{y}\right)$ (cartesian components), describing the complex slowly varying amplitudes of the electric field [42]. The right and left circularly polarized components $\left(B_{+}, B_{-}\right)$are related to the cartesian components by the relations $B_{x}=\left(B_{+}+B_{-}\right) / \sqrt{2}$ and $B_{y}=\left(B_{+}-B_{-}\right) / i \sqrt{2}$. We then obtain 
the coupled equations describing the dynamics of the circular components after usual scaling transformations [43]

$$
\begin{aligned}
\frac{\partial B_{+}}{\partial T} & =(1+i \alpha) B_{+}+(1+i \beta) \nabla^{2} B_{+}+(1+i \varepsilon)\left|B_{+}\right|^{2} B_{+}+(1+i \mu) \nu\left|B_{+}\right|^{4} B_{+}+\left(\gamma_{r}+i \gamma_{i}\right)\left|B_{-}\right|^{2} B_{+} \\
& +\left(\delta_{r}+i \delta_{i}\right)\left|B_{-}\right|^{4} B_{+}+2\left(\delta_{r}+i \delta_{i}\right)\left|B_{-}\right|^{2}\left|B_{+}\right|^{2} B_{+},
\end{aligned}
$$

$$
\begin{aligned}
\frac{\partial B_{-}}{\partial T} & =(1+i \alpha) B_{-}+(1+i \beta) \nabla^{2} B_{-}+(1+i \varepsilon)\left|B_{-}\right|^{2} B_{-}+(1+i \mu) \nu\left|B_{-}\right|^{4} B_{-}+\left(\gamma_{r}+i \gamma_{i}\right)\left|B_{+}\right|^{2} B_{-} \\
& +\left(\delta_{r}+i \delta_{i}\right)\left|B_{+}\right|^{4} B_{-}+2\left(\delta_{r}+i \delta_{i}\right)\left|B_{-}\right|^{2}\left|B_{+}\right|^{2} B_{-},
\end{aligned}
$$

where

$$
\begin{aligned}
& \alpha=-c_{1 i} / c_{1 r}, \quad \beta=c_{2 i} / c_{2 r}, \quad \varepsilon=c_{3 i} / c_{3 r}, \quad \mu=c_{5 i} / c_{5 r}, \quad \gamma_{r}=\left(c_{3 r}+2 c_{4 r}\right) / c_{3 r}, \\
& \gamma_{i}=\left(c_{3 i}+2 c_{4 i}\right) / c_{3 r}, \quad \delta_{r}=-\left(c_{5 r}+3 c_{6 r}\right) / 2 c_{5 r}, \quad \delta_{i}=-\left(c_{5 i}+3 c_{6 i}\right) / 2 c_{5 r},
\end{aligned}
$$

with $\nu=\operatorname{sign}\left(c_{5 r} / c_{3 r}^{2}\right),(X, Y)=\sqrt{c_{2 r}}(X, Y), \quad B_{ \pm}=B_{ \pm} / \sqrt{c_{3 r}}$. In Eq. (13), $\nabla^{2} B_{+}$and $\nabla^{2} B_{-}$ represent a two-dimensional Laplacian operator describing diffraction in the transverse $(X$, $Y)$ plane, $\left|B_{+}\right|^{2} B_{+}$and $\left|B_{-}\right|^{2} B_{-}$denote the cubic self-phase modulation (SPM), $\left|B_{-}\right|^{2} B_{+}$and $\left|B_{+}\right|^{2} B_{-}$correspond to the cubic cross-phase modulation (XPM), $\left|B_{+}\right|{ }^{4} B_{+}$and $\left|B_{-}\right|^{2} B_{-}$denote the quintic SPM, $\left|B_{-}\right|^{2}\left|B_{+}\right|^{2} B_{+}$and $\left|B_{+}\right|^{2}\left|B_{-}\right|^{2} B_{-}$represent the mixed quintic XPM, and $\left|B_{-}\right|^{4} B_{+}$and $\left|B_{+}\right|^{4} B_{-}$denote the quintic XPM. In the following $\delta, \beta, \varepsilon, \mu, \gamma_{r}, \gamma_{i}, \delta_{r}$, and $\delta_{i}$ are real parameters of SPM and XPM terms of Eq. (13). $\alpha$ is related to the linear loss $(\alpha<0)$ or gain $(\alpha>0)$. $\beta$ is related to the strength of diffraction, and $\varepsilon$ to the nonlinear frequency detuning. $\mu$ stands for the saturation of the nonlinear frequency detuning, $\gamma_{r}$ and $\gamma_{i}$ are the nonlinear cross coefficients related to the cubic XPM, $\delta_{r}$ and $\delta_{i}$ are the nonlinear cross coefficients related to the quintic XPM, $\nu$ represents the nonlinear coefficient related to the quintic SPM.

\section{Modulational instability: Linear analysis}

MI constitutes one of the most fundamental effects associated with wave propagation in nonlinear media. It signifies the exponential growth of a weak perturbation of the wave as it propagates. The gain leads to the amplification of sidebands, which break up the otherwise uniform wave and generate fine localized structures. Thus, it may act as a precursor for the 
240

241

formation of bright solitons. In order to study the MI of Eqs.(13a) and (13b), describing the dynamics of the circular components, we use the standard linear stability analysis. In doing so, we consider the propagation of the exact continuous-wave solutions of Eqs.(13a) and (13b) to take the form of two plane waves [39]

$$
B_{+}=M \exp \left\{i\left(k_{1} X+l_{1} Y-w_{1} T\right\},\right.
$$

$$
B_{-}=P \exp \left\{i\left(k_{2} X+l_{2} Y-w_{2} T\right\},\right.
$$

where the positive real numbers $M$ and $P$ represent the amplitudes of waves $B_{+}(X, Y, T)$ and $B_{-}(X, Y, T)$, respectively. $w_{1}$ and $w_{2}$ are real numbers representing the angular frequencies. The wave vectors are represented by real numbers $k_{1}, k_{2}, l_{1}$, and $l_{2}$. The substitution of plane wave solutions $B_{+}$and $B_{-}$into the system of the coupled cubic-quintic CGLE Eqs. (13a) and (13b) leads to a set of four equations (requiring both imaginary and real parts to be zero), representing the dispersion relations

$$
1-\left(k_{1}^{2}+l_{1}^{2}\right)+\left(1+\nu M^{2}\right) M^{2}+\left(\gamma_{r}+\frac{1}{2} \delta_{r} P^{2}\right) P^{2}+\delta_{r} P^{2} M^{2}=0,
$$

$$
1-\left(k_{2}^{2}+l_{2}^{2}\right)+\left(1+\nu P^{2}\right) P^{2}+\left(\gamma_{r}+\frac{1}{2} \delta_{r} M^{2}\right) M^{2}+\delta_{r} P^{2} M^{2}=0,
$$

$$
w_{1}=\nu \mu M^{4}-\left(\varepsilon+P^{2} \delta_{i}\right) M^{2}-\frac{1}{2} P^{4} \delta_{i}-\alpha+\beta\left(k_{1}^{2}+l_{1}^{2}\right)-\gamma_{i} P^{2},
$$

$$
w_{2}=\nu \mu P^{4}-\left(\varepsilon+M^{2} \delta_{i}\right) P^{2}-\frac{1}{2} M^{4} \delta_{i}-\alpha+\beta\left(k_{2}^{2}+l_{2}^{2}\right)-\gamma_{i} M^{2}
$$

Assuming that $B_{+}(X, Y, T)$ and $B_{-}(X, Y, T)$ remain space-independent during propagation inside the medium, i.e., $B_{+}=M e^{\left(-i w_{1} T\right)}$ and $B_{-}=P e^{\left(i w_{2} T\right)}$, Eqs. (16a)-(16d) are readily solved to obtain the steady-state solutions. For the case where $P=M$, it follows that

$$
M_{ \pm}=\sqrt{\frac{-\left(1+\gamma_{r}\right) \pm \sqrt{\left(1+\gamma_{r}\right)^{2}-2\left(2 \nu+3 \delta_{r}\right)}}{\left(2 \nu+3 \delta_{r}\right)}} .
$$


For $P \neq M$, and $M$ given by Eq. (17), we obtain

$$
P_{ \pm}=\sqrt{\frac{-\left(\gamma_{r}+M^{2} \delta_{r}\right) \pm \sqrt{\left(\gamma_{r}+M^{2} \delta_{r}\right)^{2}-2 \delta_{r}\left(1+P^{2}+\nu P^{4}\right)}}{\delta_{r}}},
$$

and

$$
w_{1}=-\nu \mu M^{4}-\left(\varepsilon+P^{2} \delta_{i}\right) M^{2}-\frac{1}{2} P^{4} \delta_{i}-\alpha-\gamma_{i} P^{2},
$$

$$
w_{2}=-\nu \mu P^{4}-\left(\varepsilon+M^{2} \delta_{i}\right) P^{2}-\frac{1}{2} M^{4} \delta_{i}-\alpha-\gamma_{i} M^{2} .
$$

Then, the plane wave solutions are completely defined as functions of the model parameters. Therefore, the question about the stability of these steady-state solutions arises. To have more insights, a perturbation analysis is conducted in order to verify if these solutions are stable against small perturbations. Assuming perturbations in the form

$$
B_{+}(X, Y, T)=[P+\epsilon V(X, Y, T)] e^{i\left(k_{2} X+l_{2} Y-w_{2} T\right)},
$$

$$
B_{-}(X, Y, T)=[M+\epsilon U(X, Y, T)] e^{i\left(k_{1} X+l_{1} Y-w_{1} T\right)},
$$

where $U(X, Y, T)$ and $V(X, Y, T)$ are small deviations from the stationary solutions of the right and left circular polarized components, i.e., both $|U(X, Y, T)|$ and $|V(X, Y, T)|$ are small compared to $M$ and $P$. Substituting Eqs. (20) into Eqs.(13a) and (13b), and linearizing in $U$ and $V$, we obtain that the dynamical equations for the perturbations are written as

$$
\begin{aligned}
\frac{\partial U}{\partial T}= & (1+i \beta) \nabla_{\perp}^{2} U+2(-\beta+i)\left(k_{1} \frac{\partial U}{\partial x}+l_{1} \frac{\partial U}{\partial y}\right)+(1+i \varepsilon) M^{2}\left(U+U^{*}\right) \\
& +\left(\gamma_{r}+i \gamma_{i}\right) M P\left(V+V^{*}\right)+\nu(1+i \mu) M^{4}\left(U+U^{*}\right) \\
& +2\left(\delta_{r}+i \delta_{i}\right) M^{2} P^{2}\left(U+U^{*}\right)+\left(\delta_{r}+i \delta_{i}\right)\left(M P^{3}+M^{3} P\right)\left(V+V^{*}\right)
\end{aligned}
$$


where $U^{*}\left(V^{*}\right)$ stands for the complex conjugate of the perturbation in the field amplitude. This pair of coupled complex linear equations can be solved by taking its general solutions as

$$
U(X, Y, T)=A_{1} e^{i(K X+L Y-\Omega T)}+A_{2}^{*} e^{-i\left(K X+L Y-\Omega^{*} T\right)},
$$

$$
V(X, Y, T)=B_{1} e^{i(K X+L Y-\Omega T)}+B_{2}^{*} e^{-i\left(K X+L Y-\Omega^{*} T\right)},
$$

where $K$ and $L$ are the wave numbers, $\Omega$ is the modulation frequency, $A_{1}, A_{2}, B_{1}$, and $B_{2}$ are constant complex amplitudes. By substituting Eq. (22) into the pair of coupled complex linear equations (21a) and (21b), we obtain a linear homogeneous system of equations in terms of $A_{1}$, $A_{2}, B_{1}$ and $B_{2}$, i.e.,

$$
H \times\left(\begin{array}{llll}
A_{1} & A_{2} & B_{1} & B_{2}
\end{array}\right)^{T}=0,
$$

where the fourth-order square matrix of the system is given by

$$
H=\left(\begin{array}{cccc}
\left(n_{11}+i \Omega\right) & n_{12} & n_{13} & n_{12} \\
n_{21} & \left(n_{23}+i \Omega\right) & n_{22} & n_{22} \\
n_{12} & n_{12} & \left(n_{32}+i \Omega\right) & n_{34} \\
n_{22} & n_{22} & n_{42} & \left(n_{44}+i \Omega\right)
\end{array}\right)
$$

with the matrix elements $n_{i, j}(i, j=1,2,3,4)$ being

$$
\begin{aligned}
& n_{11}=-(1+i \beta)\left(l^{2}+k^{2}+2\left(L l_{1}+K k_{1}\right)\right)+(1+i \varepsilon) M^{2}+2 \nu(1+i \mu) M^{4}+2\left(\delta_{r}+i \delta_{i}\right) M^{2} P^{2} \\
& n_{12}=\left(\gamma_{r}+i \gamma_{i}\right) M P+2\left(\delta_{r}+i \delta_{i}\right)\left(M P^{3}+M^{3} P\right), \\
& n_{13}=(1+i \varepsilon) M^{2}+2 \nu(1+i \mu) M^{4}+2\left(\delta_{r}+i \delta_{i}\right) M^{2} P^{2}, \\
& n_{21}=(1-i \varepsilon) M^{2}+2 \nu(1-i \mu) M^{4}+2\left(\delta_{r}-i \delta_{i}\right) M^{2} P^{2}, \\
& n_{22}=\left(\gamma_{r}-i \gamma_{i}\right) M P+2\left(\delta_{r}-i \delta_{i}\right)\left(M P^{3}+M^{3} P\right), \\
& n_{23}=-(1-i \beta)\left(l^{2}+k^{2}-2\left(L l_{1}+K k_{1}\right)\right)+(1-i \varepsilon) M^{2}+2 \nu(1-i \mu) M^{4}+2\left(\delta_{r}-i \delta_{i}\right) M^{2} P^{2}, \\
& n_{32}=-(1+i \beta)\left(l^{2}+k^{2}+2\left(L l_{2}+K k_{2}\right)\right)+(1+i \varepsilon) P^{2}+2 \nu(1+i \mu) P^{4}+2\left(\delta_{r}+i \delta_{i}\right) M^{2} P^{2}, \\
& n_{34}=(1+i \varepsilon) P^{2}+2 \nu(1+i \mu) P^{4}+2\left(\delta_{r}+i \delta_{i}\right) M^{2} P^{2}, \\
& n_{42}=(1-i \varepsilon) P^{2}+2 \nu(1-i \mu) P^{4}+2\left(\delta_{r}-i \delta_{i}\right) M^{2} P^{2}, \\
& n_{44}=-(1-i \beta)\left(l^{2}+k^{2}-2\left(L l_{2}+K k_{2}\right)\right)+(1-i \varepsilon) P^{2}+2 \nu(1-i \mu) P^{4}+2\left(\delta_{r}-i \delta_{i}\right) M^{2} P^{2} .
\end{aligned}
$$

The nontrivial solutions of this system require that $\operatorname{Det}(H)=0$, which leads to the nonlinear dispersion relation

$$
\Omega^{4}+C \Omega^{3}+D \Omega^{2}+E \Omega+F=0
$$


where

$$
\begin{aligned}
C= & -I\left(n_{32}+n_{11}+n_{23}+n_{44}\right), \\
D= & -n_{32}\left(n_{44}+n_{11}\right)-n_{23}\left(n_{32}+n_{11}+n_{44}\right)+\left(n_{12}+n_{22}\right)^{2}+n_{34} n_{42}+n_{21} n_{13}-n_{44} n_{11}, \\
E= & I n_{22}^{2}\left(n_{34}+n_{13}-n_{32}-n_{11}\right)+I n_{12}^{2}\left(n_{42}+n_{21}-n_{44}-n_{23}\right)+I n_{12} n_{22}\left(n_{21}+n_{13}+n_{34}\right. \\
& \left.+n_{42}-n_{11}-n_{23}-n_{44}-n_{32}\right)+I n_{11}\left(n_{44} n_{23}+n_{32} n_{23}+n_{44} n_{32}-n_{42} n_{34}\right) \\
& -I n_{21} n_{13}\left(n_{44}+n_{32}\right)+I n_{23}\left(n_{44} n_{32}-n_{34} n_{42}\right), \\
F= & n_{11}\left(n_{22}^{2}\left(n_{34}-n_{32}\right)+n_{12} n_{22}\left(n_{42}-n_{44}\right)+n_{23}\left(n_{44} n_{32}-n_{34} n_{42}\right)+n_{23}\left(n_{32} n_{44}-n_{43} n_{42}\right)\right) \\
& +n_{12}^{2}\left(n_{44}\left(n_{21}-n_{23}\right)+n_{42}\left(n_{23}-n_{21}\right)\right)+n_{12}\left(n_{22} n_{23}\left(n_{34}-n_{32}\right)+n_{22}\left(n_{21}\left(n_{32}-n_{34}\right)\right.\right. \\
& \left.\left.+n_{13}\left(n_{44}-n_{42}\right)\right)\right)+n_{13} n_{22}^{2}\left(n_{32}-n_{34}\right)+n_{21} n_{13}\left(n_{34} n_{42}-n_{32} n_{44}\right) .
\end{aligned}
$$

It is obvious that the coefficients in Eq. (25) are complex, and are functions of the wave vectors, amplitudes and system parameters, respectively. It is important to mention that an equation similar to Eq. (25) was obtained by Gholam-Ali et al. [39]. Nevertheless, one should note that our results encompasses theirs, due to the presence of additional terms brought by the model under consideration. These new terms are proportional to the complex parameters $\left(\delta_{r}+i \delta_{i}\right)$ in the relation defining the quantities $n_{j k}(j, k=1, \ldots, 4)$ of the $4 \times 4$ complex matrix $H$. From the solutions of the dispersion relation, we investigate about the stability of the steady-state solutions by determining the MI gain. In that direction, the roots of the dispersion relation (25) are found to be

$$
\Omega_{+}^{ \pm}= \pm \frac{1}{2} \sqrt{\frac{-C^{3}+4 C D-8 E}{4 P_{2}}+\frac{3 C^{2}}{4}-2 D-p_{2}^{2}}-\frac{C}{4}+\frac{p_{2}}{2},
$$

$$
\Omega_{-}^{ \pm}= \pm \frac{1}{2} \sqrt{-\frac{-C^{3}+4 C D-8 E}{4 P_{2}}+\frac{3 C^{2}}{4}-2 D-p_{2}^{2}}-\frac{C}{4}-\frac{p_{2}}{2},
$$

where $P_{2}=\sqrt{\frac{C^{2}}{4}+\frac{-3 C E+D^{2}+12 F}{3 P_{1}}-\frac{2 D-p_{1}}{3}}, p_{1}=2^{-1 / 3} \sqrt[3]{p_{0}+27 C^{2} F-9 C D E+2 D^{3}-72 D F+27 E^{3}}$, and $p_{0}=\sqrt{\left(27 C^{2} F-9 C D E+2 D^{3}-72 D F+27 E\right)^{2}-4\left(-3 C E+D^{2}+12 F\right)^{3}}$.

As it is obvious, the quantities $\Omega_{ \pm}^{+}$and $\Omega_{ \pm}^{-}$depend on the laser cavity parameters that are included in the dispersion relation coefficients. MI occurs only when at least one of the eigenvalues of the stability matrix $H$ possesses a nonzero and negative imaginary part, which results in an exponential growth of the amplitude with the perturbation. MI is measured by the power gain and is defined as

$$
G_{ \pm}^{ \pm}=2 \operatorname{Im}\left(\Omega_{ \pm}^{ \pm}\right)>0
$$

where $\operatorname{Im}\left(\Omega_{ \pm}^{ \pm}\right)$denotes the imaginary part of $\left.\Omega_{ \pm}^{ \pm}\right)[39,44]$. 
(a)

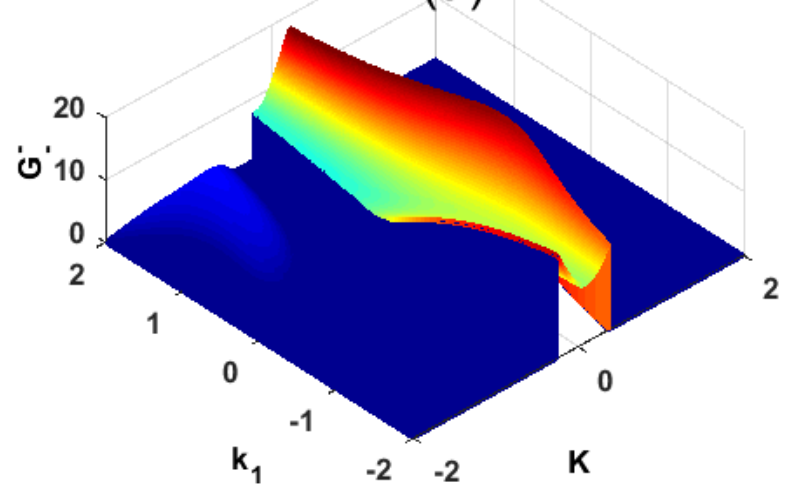

(b)

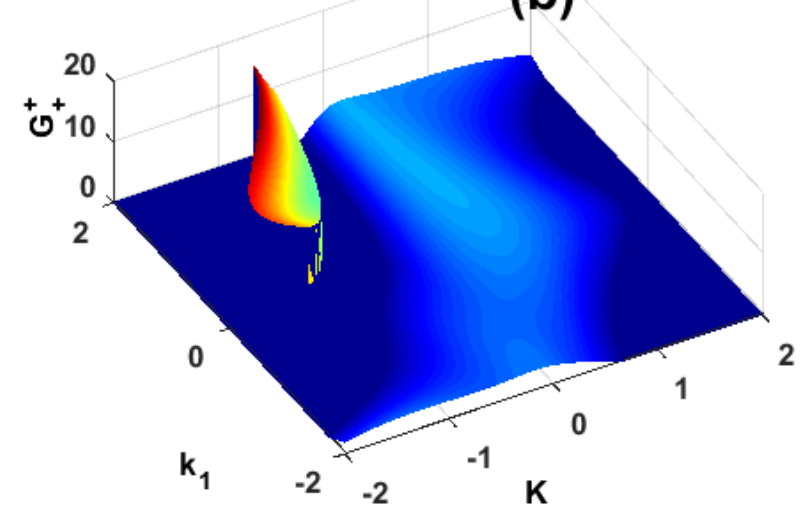

Figure 1: (Color online) Gain spectrum versus perturbed wavenumber $K$ and non perturbed wavenumber $k_{1}$, at $l_{1}=10.20 ; l_{2}=10 ; k_{2}=1$, and $L=0.4$.

\section{MI Analysis}

Considering the MI gain (see Eq. (22)), several qualitative situations emerge depending on the system parameters. The regions of instability are called MI gain spectra and this takes place when $G_{-}^{-}=2 \operatorname{Im}\left(\Omega_{-}^{-}\right)>0$, or $G_{-}^{+}=2 \operatorname{Im}\left(\Omega_{-}^{+}\right)>0$, or $G_{+}^{-}=2 \operatorname{Im}\left(\Omega_{+}^{-}\right)>0$, or $G_{+}^{+}=$ $2 \operatorname{Im}\left(\Omega_{+}^{+}\right)>0$. It is well-known that pattern formation may take place in the cubic-quintic CGL equation when the gain/loss and diffraction/nonlinearities are well balanced. We start our investigation by analysing the influence of wavenumbers $K$ and $k_{1}$ on the MI. We consider the following parameters of MB equation, $\gamma_{\perp}=9.9 \times 10^{10} s^{-1}, \gamma_{\|}=3 \times 10^{7} s^{-1}, w_{a}=0.52 \times 10^{9} s^{-1}$, $\kappa=6.9 \times 10^{7}, \tilde{D}_{0}=12.5$ and the lasing wavelength $\lambda=10.6 \mu m$ [40], Fig. 1 shows the dependence of the gain $\mathrm{G}$ with respect to the wavenumbers $K$ and $k_{1}$.

Figs. 1(a) and (b), describe the MI process given by $\Omega_{+}^{+}$(see 26(a)) and $\Omega_{-}^{-}$(see 26(b)), respectively . Fig. 1(a) shows the MI process around the perturbed wavenumber $K=0$, and in Fig. 1(b), the maximum instability is isolated in the domains $K<0$ and $k_{1}>0$, the second domain of hight intensity, but lower than the preceding isolate MI domain for the same figure is observed around $K=0$. Fig. 2 shows more insight the manifestation of the MI when increasing the value of $w_{a}$.

Figs. 2 (a) and 2 (c) correspond to the MI gain spectrum related to $\Omega_{-}^{-}$, and Figs. 2 (b) and $2(\mathrm{~d})$ correspond to $\Omega_{+}^{+}$. Figures 2 (a) and 2 (b) are obtained for $w_{a}=0.34 \times 10^{9} s^{-1}$, Fig. 2 (a), in particular seems similar to Fig. 1 (a), while the edge and the width of the sidelobe 

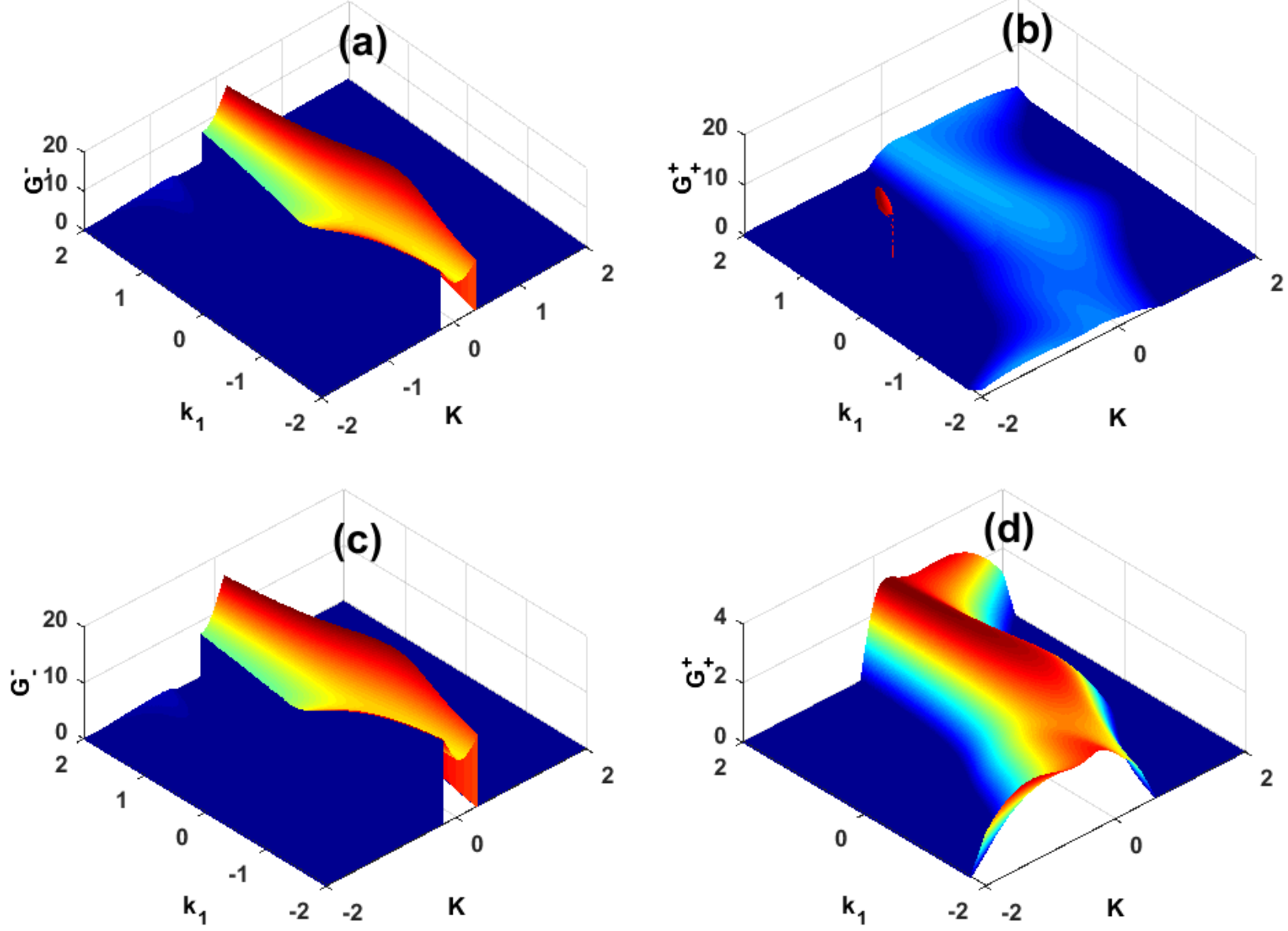

Figure 2: (Color online) First line Gain spectrum related to the solution $\Omega_{-}^{-}$and $\Omega_{+}^{+}$in the second line, versus the perturbed wavenumber $K$ and the wavenumber $k_{1}$ at $\kappa=6.9 \times 10^{7}$, $\gamma_{\perp}=3.9 \times 10^{10}, \gamma_{\|}=3 \times 10^{7}$. (a) and (b) $w_{a}=0.34 \times 10^{9}$, (c) and (d) $w_{a}=0.52 \times 10^{9}$. 
(a)
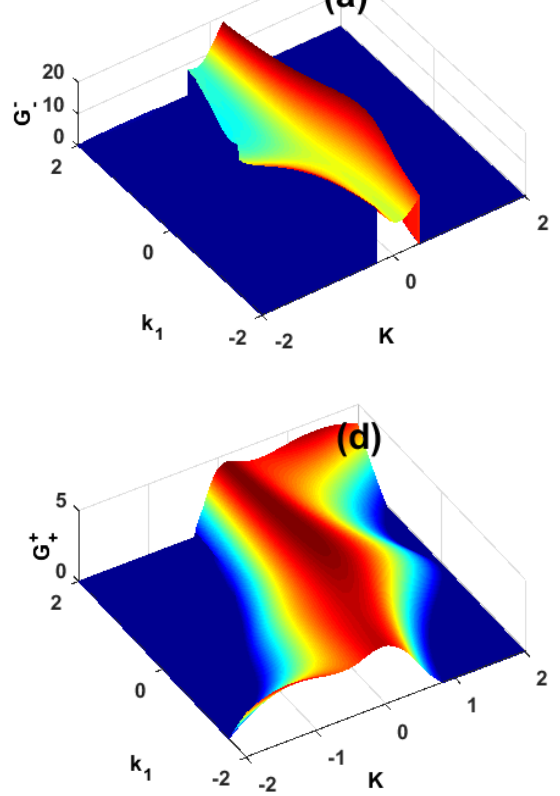

(b)
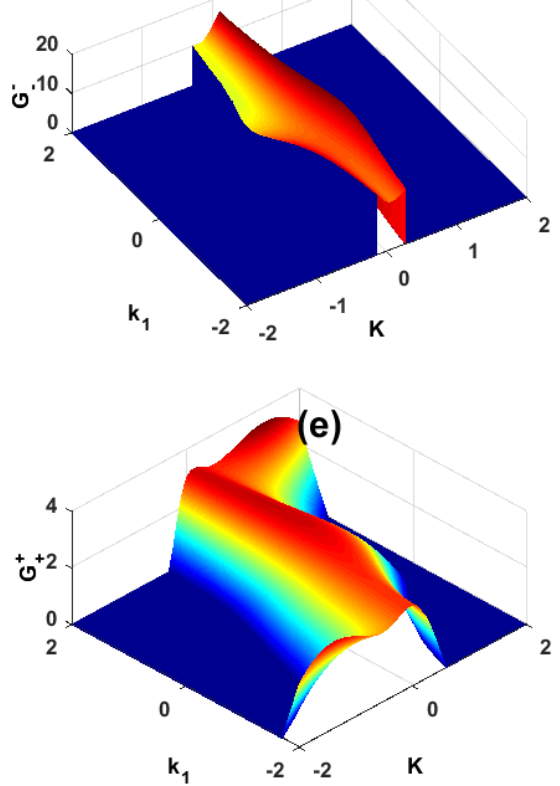

(c)
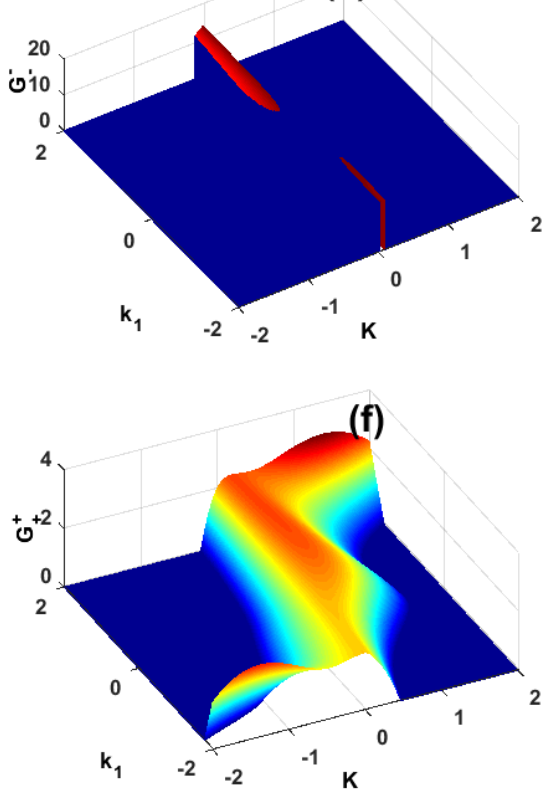

Figure 3: Color online) first line Gain spectrum related to the solution $\Omega_{-}^{-}$and $\Omega_{+}^{+}$in the second line, versus the perturbed wavenumber $K$ and the wavenumber $k_{1}$ at $w_{a}=0.52 \times 10^{9}$, $\gamma_{\perp}=3.9 \times 10^{10}, \gamma_{\|}=3 \times 10^{7}$. (a) and (d) $\kappa=6.9 \times 10^{7}$, (b) and (e) $\kappa=4.9 \times 10^{7}$, and (c) and (e) $\kappa=4.1 \times 10^{7}$.

observed in Fig. 1 (b), have been considerably reduced keeping the same intensity (see Fig 2 (b)). On the other hand, when slightly increasing the value of $w_{a}$ to $0.52 \times 10^{9}$, we observe in Fig. 2 (d), the disappearance of the sidelobe obtained in Fig. 2 (b) and Fig. 1 (b). Also, the intensity of the MI gain of Fig. 2 (d) has decreased in comparison to Figs. 1 (b) and Figs. 2(b), and more importantly, unstable waves may be expected for $0.5 \leq w_{a} \leq w_{\text {acr }}$.

Next, we analyze the influence of the cavity damping coefficient $\kappa$ on the MI. Here, we keep the rest of the parameters of the MB equation the same as in Figs. 2 (c) and (d), while varying the cavity damping coefficient $\kappa$. Fig. 3 shows the gain spectrum for $\kappa=6.9 \times 10^{7} s^{-1}$, $\kappa=4.9 \times 10^{7} s^{-1}$ and $\kappa=4.1 \times 10^{7} s^{-1}$, respectively, with $\gamma_{\perp}=3.9 \times 10^{10} s^{-1}$.

In Figs. 3(a) - (c), the gain spectrum is symmetric with respect to the line $K=0$ and the MI gain decreases with the decreasing of $\kappa$, and collapses for $0.41<\kappa<\kappa_{c r}$. Despite the decreasing of the width of the MI gain $G_{-}^{-}$, its intensity remains constant when decreasing the value of $\kappa$. Figs. 3(d) - (f) describe the evolution of the MI gain when $\kappa$ decreases. Contrary to the MI gain spectrum $G_{-}^{-}$, the gain spectrum $G_{+}^{+}$evolves with low intensity, and it also decreases when $\kappa$ decreases. The same remark is made on its width (see Figs. 3(d)-(f)). As a 
whole, we have noted the disappearance of the MI gain spectrum for $\kappa<4 \times 10^{7} s^{-1}$. Otherwise, for $\kappa=4.9 \times 10^{7} s^{-1}$, we have observed that when increasing $\gamma_{\|}$, the MI gain spectrum also disappears at $\gamma_{\|} \simeq 3.9 \times 10^{7} s^{-1}$. This suitably agree with the fact that in class B laser, $\gamma_{\perp}>>\kappa>\gamma_{\|}[28,40]$

So, to enhance the MI gain, we can adjust the system in order for its parameters to correspond to a specific class of laser, such as class B laser which is the concern of this work. They can bring about new sidebands, shift the existing sidebands, or merge them.

As noticed in this analysis, laser parameters are very influential to the occurrence of MI, especially when plane wave parameters fall well inside the instability domain. Under such conditions, the plane wave solution will be said to be unstable under modulation, and the direct consequence will be its disintegration into nonlinear wave patterns. Otherwise, wave modulation will not take place, since the choice of parameters will not be favorable to a suitable balance between gain/loos and diffractive/nonlinear effects.

\section{$5 \quad$ Numerical simulations}

To further study the MI phenomena, numerical simulations are reported in this section. They are in fact used to check the accuracy of our analytical predictions. Computer simulations are performed using the laser $(2+1) \mathrm{D}$ vectorial cubic-quintic CGL Eq. (13) by means of the split-step Fourier Method, with a time-step $\Delta T=10^{-3}$, on a mesh of size $100 \times 100$, with space-steps $\Delta X=\Delta Y=0.01$ and fixed boundary conditions at the edges of the domain. The used initial conditions are slightly modulated plane waves given by [32]

$$
B_{-}(X, Y, T=0)=M\left[1+A_{m} \sin (2 \pi(K X+L Y))\right] e^{-i\left(k_{1} X+l_{1} Y\right)},
$$

$$
B_{+}(X, Y, T=0)=P\left[1+A_{P} \sin (2 \pi(K X+L Y))\right] e^{-i\left(k_{2} X+l_{2} Y\right)},
$$

where $A_{m}$ and $A_{p}$ are amplitude modulations, $K$ and $L$ are the frequencies of weak sinusoidal modulations imposed on the continuous waves, in the $X$ and $Y$ directions, respectively.

For the rest, we consider the competiting effects between of cubic and quintic terms based on the laser parameters. From various numerical examples, we note that when $\kappa>\gamma_{\|}$, the 

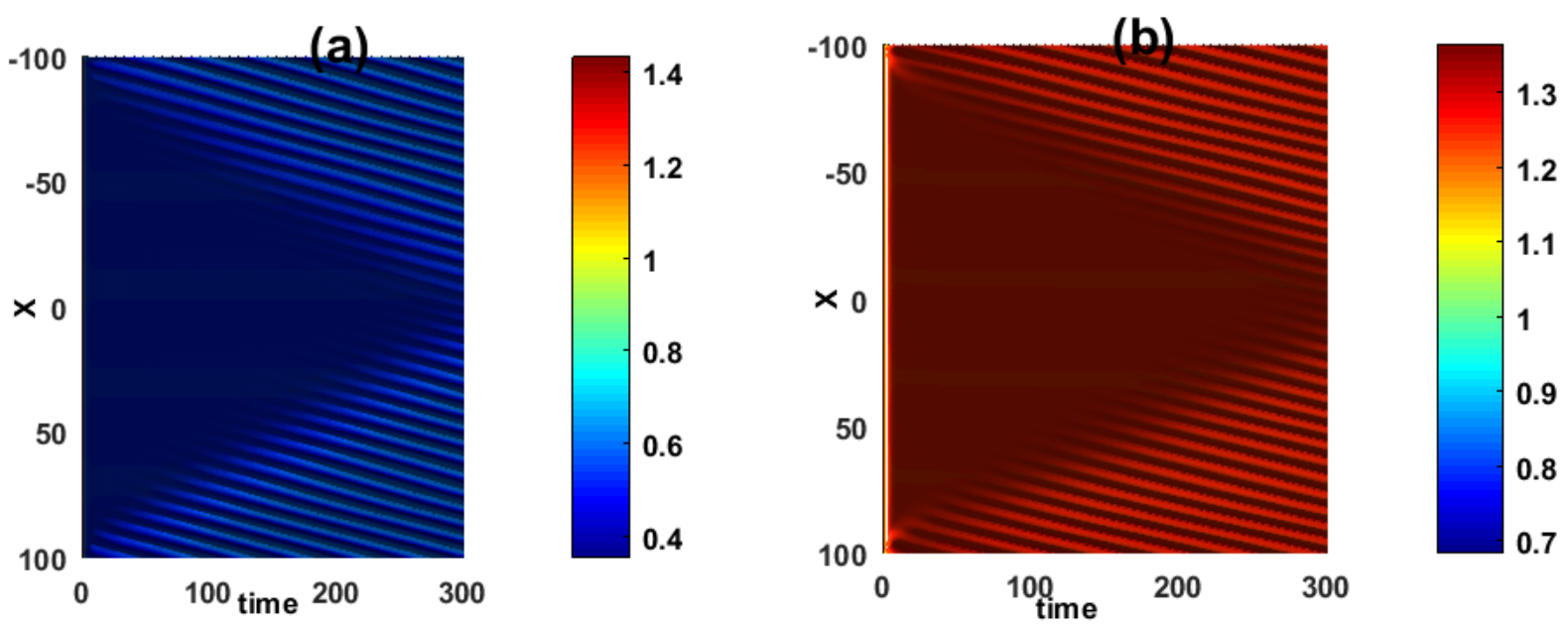

Figure 4: Panels (a) and (b) show the evolution of a plane wave leading to chaotic behavior at $\alpha=-0.0147, \beta=0.0147, \varepsilon=0.0267, \gamma_{r}=1.4892, \gamma_{i}=0.0147, \mu=0.0267, \delta_{r}=-0.5013$, and $\delta_{i}=0.0170$.

outcome is the generation of periodic of nonlinear structures, due to the competition between loss and gain, diffraction and nonlinearity. However, when we neglect the quintic nonlinear term [28], the system does not show any propagation, but collapses after laps time of propagation. However, keeping only the quintic nonlinearity term, we obtain the generation of incoherent patterns.

Fig. 4 shows the space-time plot of a continuous wave, under MI, using the parameters $w_{a}=0.52 \times 10^{9} s^{-1}, \gamma_{\perp}=3.9 \times 10^{10} s^{-1}, \gamma_{\|}=3.85 \times 10^{7} s^{-1}$, and $\kappa=4.9 \times 10^{7} s^{-1}$. The related dissipation parameters are given in the figure caption. MI manifests itself with the appearance of new instability features, where Figs. 4(a) and (b) show the spatiotemporal evolution of $B_{-}(X, 0, T)$ and $B_{+}(X, 0, T)$, respectively, represented by their corresponding density plots. In two dimensions, the phenomenon is more ostensible as shown in Fig. 5, at different times, where panels Figs. 5 (a)-(d) are obtained at the respective times $T=0, T=400, T=2000$ and $T=10000$. There is, in fact, an exponential decrease of the wave amplitudes at the initial time of propagation, followed by an important explosion of wave amplitudes, with lower intensities. This, in fact, corroborates our predictions, which, due to the chosen parameter values from the gain spectrum (see Fig. 3(e)), lead to the disintegration of the initial plane wave solutions.

Figure 6 reveals more insight into the effect of the relaxation rate $\gamma_{\perp}$ and $\gamma_{\|}$for the polarization and the population inversion, respectively, the atomic frequency $w_{a}$ and the cavity damping coefficient $\kappa$, on the generation of nonlinear structures. The following experimental 
(a)

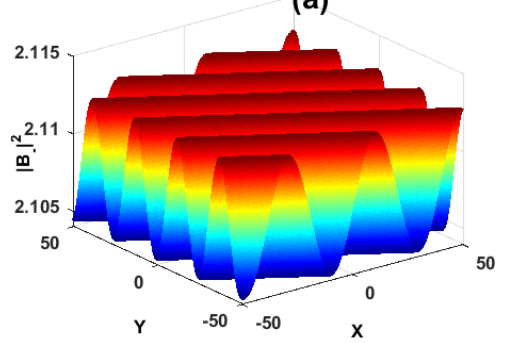

(e)

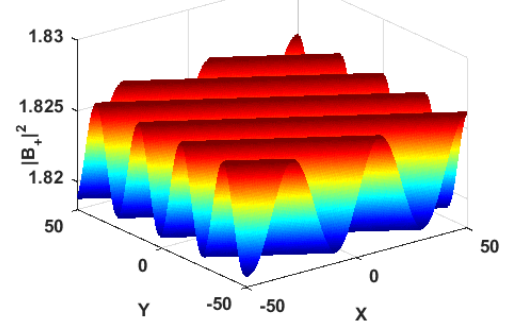

(b)

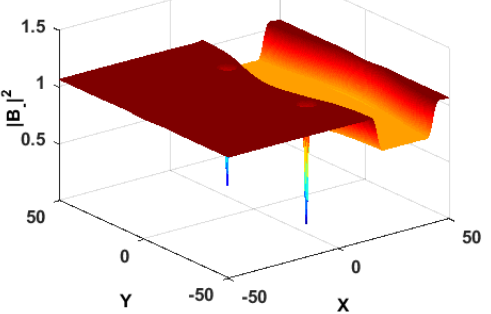

(f)

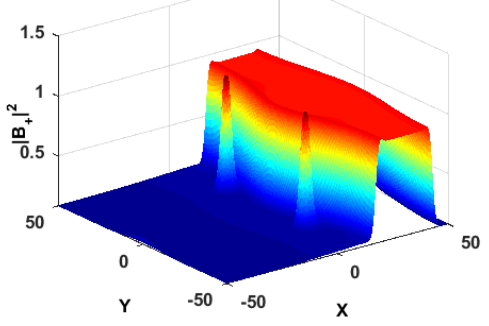

(c)

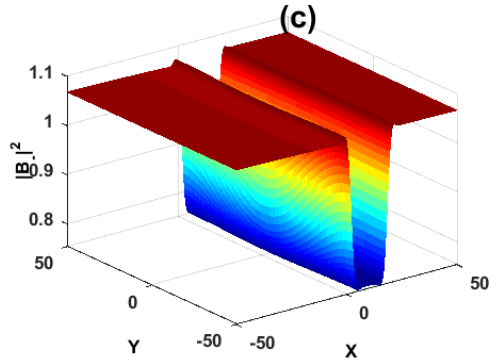

(g)

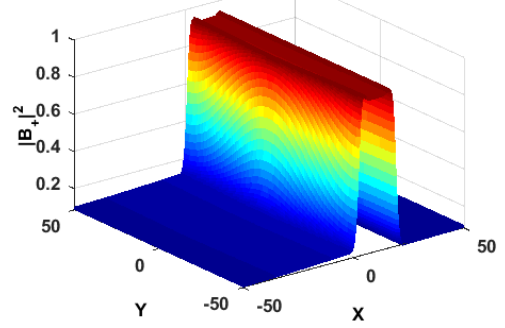

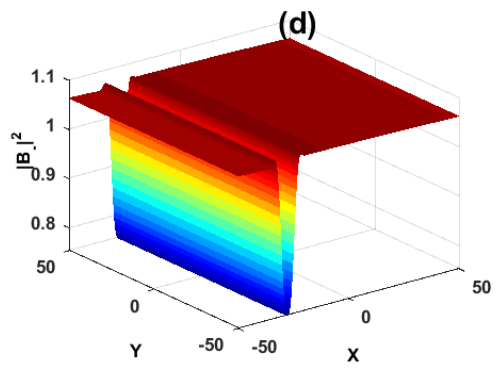

(h)

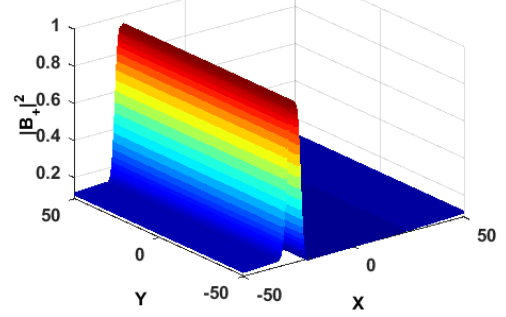

Figure 5: Two-dimensional manifestation of MI at different instants: (a) and (e) $T=2$, (b) and (f) $T=400,(\mathrm{c})$ and (g) $T=2000$, (d) and (h) $T=10000$. (a) to (d) correspond to $B_{-}$, (e) to (h) correspond to $B_{+}$.

parameter values have been used [40]: $\lambda=10.6 \mu m, \gamma_{\perp}=3.95 \times 10^{9} s^{-1}, \gamma_{\|}=4.5 \times 10^{6} s^{-1}$, $w_{a}=2.8 \times 10^{8} s^{-1}$, linear loss $\alpha=-0.0401$, diffusion parameter $\beta=0.0401$, cubic nonlinear gain $\varepsilon=0.0267$, quintic nonlinear loss $\mu=-0.0267$, cubic coupled parameters $\gamma_{r}=1.3952$ and $\gamma_{i}=0.0148$, and quintic coupled parameters $\delta_{r}=-0.5013$ and $\delta_{i}=0.0176$

In comparison to Fig. 5, we have increased the photon lifetime $\tau=\frac{1}{\gamma_{\|}} s$ inside the cavity to plot Fig. 6. Beyond some parameter change, the features at Fig. 6 are similar to those in Fig. 5. However, we realize in Figs. 6 (a)-(d), and Figs. 6 (e)-(h), that during the propagation, the wave amplitude initially decreases. Thereafter, we observe a stage of constant amplitude with patterns, that ends by aperiodic wave structures. Here, we note that when the soliton becomes stable, the two wave behave in phases, with the same intensity. As mentioned at the beginning of this section, the class B laser is described by three- or four- level atomic schemes, where the extra levels are necessary for obtaining population inversion with large lifetime of population inversion, which is a necessary condition to for amplification and lasing [45]. Obviously, the propagation of the soliton is altered. Moreover, when the quintic nonlinearity (quintic loss and coupled) is not taken into account, the soliton amplitude abruptly and exponentially decreases under MI and does not show any change. So, in order for stable periodic soliton to form, all terms in Eq. (13) should be considered. 
(a)

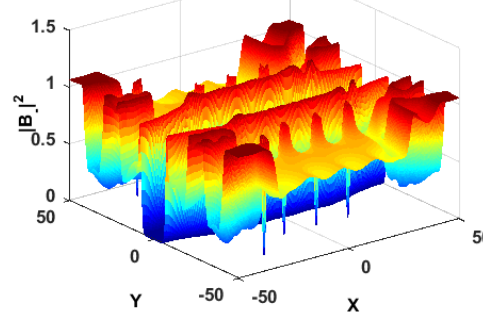

(e)

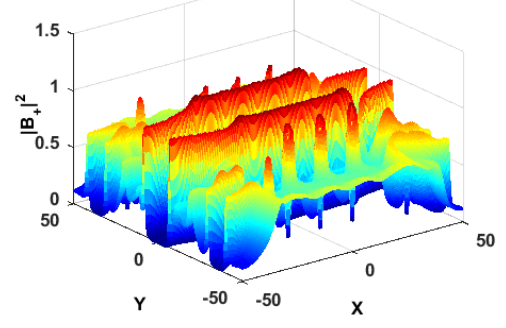

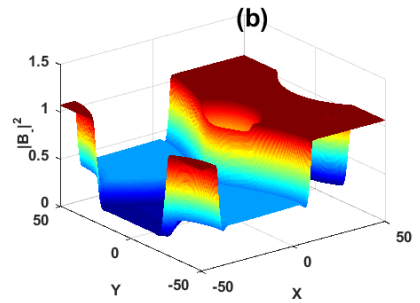

(f)

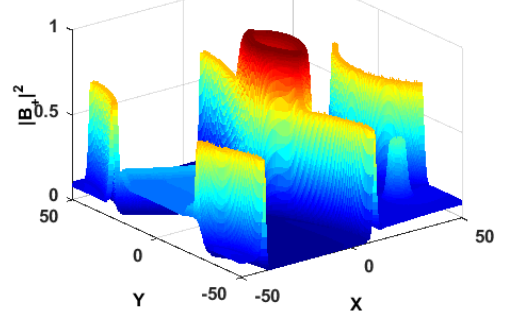

(c)

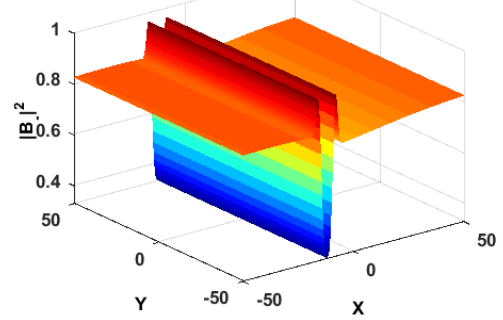

(g)

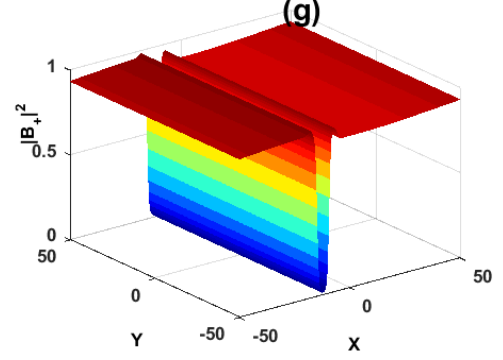

(d)

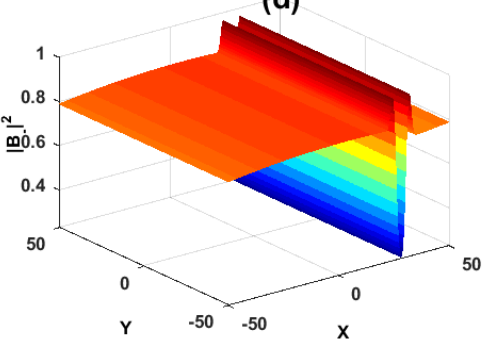

(h)

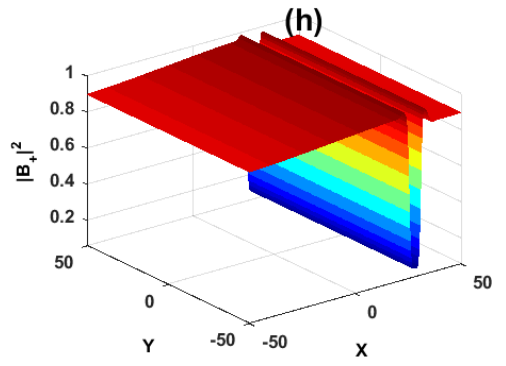

Figure 6: Two-dimensional manifestation of MI at different instants: (a) and (e) $T=25$, (b) and (f) $T=400,(\mathrm{c})$ and (g) $T=5000$, (d) and (h) $T=50000$. (a) to (d) correspond to $B_{-}$, (e) to (h) correspond to $B_{+}$.

In general, the balance between gain/loss and diffraction/nonlinearities gives rise to plane wave disintegration, main characteristics of MI. This leads to localized modes that display interesting and new behaviors, related to the change in the laser cavity parameters. This means that with more choices of suitable parameters, the system might display more exotic behaviors under the activation of the MI.

\section{Conclusion}

In summary, the first achievement of the present work was the successful derivation of the $(3+1)$ D vectorial cubic-quintic complex GL equation, modeling the interaction of an electromagnetic field with matter in a laser, near the lasing threshold. Then, in the second one, we used the linear stability analysis to find the instability criteria and growth rate of instability from which we got regions of parameters where wave patterns can emerge in the studied model. This has been followed by direct numerical simulation, on the generic model, in order to confirm our analytical predictions. A good agreement between the two approaches has been obtained, especially the disintegration of the plane wave solutions into nonlinear waves patterns. 


\section{${ }_{397}$ Acknowledgments}

398 The authors would like to thank the CETIC (University of Yaoundé I, Cameroon) for their 399 helpful support. CBT has received research grant from the Botswana International University 400 of Science and Technology, under the grant DVC/RDI/2/1/16I (25). CBT also thanks the 401 Kavli Institute for Theoretical Physics (KITP), University of California Santa Barbara (USA), 402 where this work was supported in part by the National Science Foundation, Grant no.NSF 403 PHY-1748958. 
tion, we have obtained:

$$
\begin{aligned}
& \mathbf{E}=\sum_{j=1}^{\infty} \epsilon^{j} \sum_{n=-j}^{+j} \mathbf{E}_{j}^{n}(r) \exp (\text { inwt }), \\
& \mathbf{P}=\sum_{j=1}^{\infty} \epsilon^{j} \sum_{n=-j}^{+j} \mathbf{P}_{j}^{n}(r) \exp (\text { inwt }), \\
& D=\sum_{j=1}^{\infty} \in^{j} \sum_{n=-j}^{+j} D_{j}^{n}(r) \exp (\text { inwt }),
\end{aligned}
$$

under the conditions $\mathbf{E}_{j}^{-n}=\left(\mathbf{E}_{j}^{n}\right)^{*}, \mathbf{P}_{j}^{-n}=\left(\mathbf{P}_{j}^{n}\right)^{*}$, and $D_{j}^{-n}=\left(D_{j}^{n}\right)^{*}$. We assume that permanent electric field, that leads to

$$
\forall j>0 \text { leads to } \mathbf{E}_{j}^{0}=0 .
$$

We focus our study to the case of $E=E_{1}^{1}, D_{1}^{0}=D_{0}$ In the presence of the intense field in the system, we have $D_{0}<<\frac{2}{\hbar w_{a}}\left(E \cdot \frac{\partial P}{\partial t}\right)$. Inserting the relation of $\mathbf{P}$ and $\mathrm{D}$ given from Eq.

(24) into Eqs. (1.2)-(1.3), it comes, for any $e^{i n w_{a} t}$, the following relations:

$$
\begin{aligned}
& w_{a}\left[\left(1-n^{2}\right) w_{a}+i \gamma_{\perp}\right]\left(\epsilon \mathbf{P}_{1}^{n}+\epsilon^{2} \mathbf{P}_{2}^{n}+\epsilon^{3} \mathbf{P}_{3}^{n}+\ldots\right)=-g \sum_{p+q=n}\left(\epsilon D_{1}^{q}+\epsilon^{2} D_{2}^{q}+\ldots\right)\left(\epsilon \mathbf{E}_{1}^{p}+\epsilon^{2} \mathbf{E}_{2}^{p}+\ldots\right)(A . \\
& \left(\gamma_{\|}+i n w_{a}\right)\left(\epsilon D_{1}^{n}+\epsilon^{2} D_{2}^{n}+\ldots\right)=\frac{2 i}{\hbar} \sum_{p+q=n} q\left(\epsilon \mathbf{E}_{1}^{p}+\epsilon^{2} \mathbf{E}_{2}^{p}+. .\right)\left(\epsilon \mathbf{P}_{1}^{q}+\epsilon^{2} \mathbf{P}_{2}^{q}+\epsilon^{3} \mathbf{P}_{3}^{q}+. .\right),
\end{aligned}
$$

where $p$ and $q$ can take the negative values, and $p+q=n$. For any power of $\epsilon$, solving these equa-

$$
\begin{aligned}
\epsilon^{1}, n=0: & \mathbf{P}_{1}^{0}=0, \quad D_{1}^{0}=D_{0} . \\
\epsilon^{1}, n=1: & \mathbf{P}_{1}^{1}=\frac{1}{\mu_{0} c^{2}}\left(-1+\frac{i k}{w_{a}}\right) \mathbf{E}_{1}^{1}, \quad D_{1}^{1}=0 . \\
\epsilon^{2}, n=0: & \mathbf{P}_{2}^{0}=0, \quad D_{2}^{0}=\frac{2 i}{\hbar \gamma_{\|}}\left(\mathbf{P}_{1}^{1} \mathbf{E}_{1}^{-1}-\mathbf{P}_{1}^{-1} \mathbf{E}_{1}^{1}\right) . \\
\epsilon^{2}, n=1: & \mathbf{P}_{2}^{1}=\frac{i g}{\gamma_{\perp} w_{a}}\left(D_{1}^{0} \mathbf{E}_{1}^{1}\right), \quad D_{2}^{1}=0 .
\end{aligned}
$$

$$
\begin{array}{ll}
\epsilon^{2}, n=2: & \mathbf{P}_{2}^{2}=0, \quad D_{2}^{2}=\frac{2 i}{\hbar\left(\gamma_{\|}+2 i w_{a}\right)}\left(\mathbf{P}_{1}^{1} \mathbf{E}_{1}^{1}\right) . \\
\epsilon^{3}, n=0: & \mathbf{P}_{3}^{0}=0, \quad D_{3}^{0}=\frac{2 i}{\hbar \gamma_{\|}}\left(\mathbf{P}_{2}^{1} \mathbf{E}_{1}^{-1}-\mathbf{P}_{2}^{-1} \mathbf{E}_{1}^{1}\right) .
\end{array}
$$$$
\epsilon^{3}, n=1: \quad \mathbf{P}_{3}^{1}=\frac{i g}{\gamma_{\perp} w_{a}}\left(D_{2}^{0} \mathbf{E}_{1}^{1}+D_{2}^{2} \mathbf{E}_{1}^{-1}\right), \quad D_{3}^{1}=0 .
$$$$
\epsilon^{3}, n=2: \quad \mathbf{P}_{3}^{2}=0, \quad D_{3}^{2}=\frac{2 i}{\hbar\left(\gamma_{\|}+2 i w_{a}\right)}\left(\mathbf{P}_{2}^{1} \mathbf{E}_{1}^{1}\right) \text {. }
$$$$
\epsilon^{3}, n=3: \quad \mathbf{P}_{3}^{3}=\frac{i g}{\left(8 w_{a}-3 i \gamma_{\perp}\right)}\left(D_{2}^{2} \mathbf{E}_{1}^{1}\right), \quad D_{3}^{3}=0,
$$

with $\mathbf{P}=\epsilon \mathbf{P}_{1}+\epsilon^{2} \mathbf{P}_{2}+\epsilon^{3} \mathbf{P}_{3}$ with $\mathbf{P}_{1}=\mathbf{P}_{1}^{1}, \quad \mathbf{P}_{2}=\mathbf{P}_{2}^{1} \quad$ and $\mathbf{P}_{3}=\mathbf{P}_{3}^{1}+\mathbf{P}_{3}^{3}$.

In the following we performs the nonlinear perturbation analysis near the laser threshold by introducing a small parameter defined by $D_{0}=D_{0 C}+\epsilon^{2} \tilde{D}_{0}(\epsilon<<1),(X, Y)=\epsilon(x, y)$, $(Z, T)=\epsilon^{2}(z, t)[28]$.

$$
\left(\begin{array}{l}
\mathbf{E} \\
\partial_{t} \mathbf{E} \\
\mathbf{P} \\
\partial_{t} \mathbf{P} \\
D
\end{array}\right)=\left(\begin{array}{l}
0 \\
0 \\
0 \\
0 \\
D_{0}
\end{array}\right)+\epsilon\left(\begin{array}{l}
\mathbf{E}_{1} \\
\partial_{t} \mathbf{E}_{1} \\
\mathbf{P}_{1} \\
\partial_{t} \mathbf{P}_{1} \\
D_{1}
\end{array}\right)+\epsilon\left(\begin{array}{l}
\mathbf{E}_{2} \\
\partial_{t} \mathbf{E}_{2} \\
\mathbf{P}_{2} \\
\partial_{t} \mathbf{P}_{2} \\
D_{2}
\end{array}\right)+\ldots
$$


with

$$
\left(\begin{array}{l}
\mathbf{E}_{1} \\
\partial_{t} \mathbf{E}_{1} \\
P_{1} \\
\partial_{t} \mathbf{P}_{1} \\
D_{1}
\end{array}\right)=\left(\begin{array}{l}
A \\
i w_{a} \mathbf{A} \\
\frac{1}{\mu_{0} c^{2}}\left(-1+\frac{i k}{w_{a}}\right) \mathbf{A} \\
\frac{i w_{a}}{\mu_{0} c^{2}}\left(-1+\frac{i k}{w_{a}}\right) \mathbf{A} \\
0
\end{array}\right) \mathrm{e}^{i\left(w t-k_{c} z\right)}+\text { c.c., } A \perp \hat{Z}
$$

Moreover, from the MB equations, some algebraic manipulation yields the following solvability condition

$\kappa \frac{\partial \mathbf{E}_{1}}{\partial T}=-2 i w_{a} \frac{\partial \mathbf{E}_{1}}{\partial T}-2 i w_{a} c\left(\frac{\partial}{\partial Z}+\frac{i}{2 k_{c}} \nabla_{\perp}^{2}\right) \mathbf{E}_{1}-\mu_{0} c^{2}\left(2 \frac{\partial}{\partial T} \frac{\partial \mathbf{P}_{1}}{\partial t}\right)$,

$2 \frac{\partial}{\partial T} \frac{\partial \mathbf{P}_{1}}{\partial t}=-\gamma_{\perp} 2 \frac{\partial \mathbf{P}_{1}}{\partial T}-g\left(\tilde{D}_{0}+D_{2}\right) \mathbf{E}_{1}$,

$\frac{\partial D_{2}}{\partial t}=-\gamma_{\|} D_{2}+\frac{2}{\hbar w_{a}}\left(\mathbf{E}_{1} \cdot \frac{\partial \mathbf{P}_{1}}{\partial t}\right)$

Combining Eqs. (A. 5) and (A. 6) just gives

$\frac{\partial \mathbf{E}_{1}}{\partial T}=\frac{2 c\left(\gamma_{\perp}-i w_{a}\right)}{k-\gamma_{\perp}+2 i w_{a}}\left(\frac{\partial}{\partial Z}+\frac{i}{2 k_{c}} \nabla_{\perp}^{2}\right) \mathbf{E}_{1}+\frac{\mu_{0} c^{2} g}{k-\gamma_{\perp}+2 i w_{a}}\left(\tilde{D}_{0}+D_{2}\right) \mathbf{E}_{1}$

The nonlinearities comes from the interaction between the population inversion and the electric field. In order to analyze the higher order diffusive term in this system, the higherorder correction $\gamma_{\perp}^{2} \frac{\partial^{2} \mathbf{P}_{1}}{\partial T^{2}}$ is needed to the polarization equation Eq.(31.2)

$$
\begin{aligned}
& \kappa \frac{\partial \mathbf{E}_{1}}{\partial T}=-2 i w_{a} \frac{\partial \mathbf{E}_{1}}{\partial T}-2 i w_{a} c\left(\frac{\partial}{\partial Z}+\frac{i}{2 k_{c}} \nabla_{\perp}^{2}\right) \mathbf{E}_{1}-\mu_{0} c^{2}\left(2 \frac{\partial}{\partial T} \frac{\partial \mathbf{P}_{1}}{\partial t}\right) . \\
& 2 \frac{\partial}{\partial T} \frac{\partial \mathbf{P}_{1}}{\partial t}=-\gamma_{\perp} \frac{\partial \mathbf{P}_{1}}{\partial t}+\gamma_{\perp}^{2} \frac{\partial^{2} \mathbf{P}_{1}}{\partial T^{2}}-g\left(\tilde{D}_{0}+D_{2}\right) \mathbf{E}_{1} . \\
& \frac{\partial D_{2}}{\partial t}=-\gamma_{\|} D_{2}+\frac{2}{\hbar w_{a}}\left(\mathbf{E}_{1} \cdot \frac{\partial \mathbf{P}_{1}}{\partial t}\right) .
\end{aligned}
$$

Substituting Eq.(A.24) into Eq.(A. 23); we obtain

$$
\begin{aligned}
& \left(\kappa-\gamma_{\perp}+2 i w_{a}\right)\left[1+\frac{2 \gamma_{\perp}^{2}}{\kappa-\gamma_{\perp}+2 i w_{a}}\left(\frac{\partial}{\partial Z}+\frac{i}{2 k_{c}} \nabla_{\perp}^{2}\right)\right] \frac{\partial \mathbf{E}_{1}}{\partial T}=2 c\left(\gamma_{\perp}-i w_{a}\right)\left(\frac{\partial}{\partial Z}+\frac{i}{2 k_{c}} \nabla_{\perp}^{2}\right) \mathbf{E}_{1} \\
& +\mu_{0} c^{2} g\left(\tilde{D}_{0}+D_{2}\right) \mathbf{E}_{1} \text {. }
\end{aligned}
$$

25 Multiplying both sides of Eq. (A. 26$)$ by $\left(\left(\kappa-\gamma_{\perp}+2 i w_{a}\right)\left[1+\frac{2 \gamma_{\perp}^{2}}{\kappa-\gamma_{\perp}+2 i w_{a}}\left(\frac{\partial}{\partial Z}+\frac{i}{2 k_{c}} \nabla_{\perp}^{2}\right)\right]\right)^{-1}$ ¿26 leads to the following amplitude equation derived by Gil [28]:

$$
\frac{\partial}{\partial T} \mathbf{A}=C_{1} A+C_{2}\left(\frac{\partial}{\partial Z}+\frac{i}{2 k_{c}} \nabla_{\perp}^{2}\right) \mathbf{A}+C_{3}\left(\frac{\partial}{\partial Z}+\frac{i}{2 k_{c}} \nabla_{\perp}^{2}\right)^{2} \mathbf{A}+C_{4}\left(\mathbf{A} \cdot \mathbf{A}^{*}\right) \mathbf{A}+C_{5}(\mathbf{A} \cdot \mathbf{A}) \mathbf{A}^{*}
$$




$$
\begin{aligned}
& C_{1}=\frac{\mu_{0} c^{2} g \tilde{D}_{0}\left(\kappa-\gamma_{\perp}+2 i w_{a}\right)}{\left(\left(\kappa-\gamma_{\perp}\right)^{2}+4 w_{a}^{2}\right)}, \\
& C_{2}=-\frac{2 c\left(\gamma_{\perp}\left(\gamma_{\perp}-\kappa\right)+2 w_{a}^{2}+i w_{a}\left(\kappa-3 \gamma_{\perp}\right)\right)}{\left(\left(\kappa-\gamma_{\perp}\right)^{2}+4 w_{a}^{2}\right)}, \\
& C_{3}=-\frac{4 c^{2} \gamma_{\perp}\left(\gamma_{\perp}^{2}\left(2 \kappa-\gamma_{\perp}\right)+\kappa\left(\kappa \gamma_{\perp}-4 w_{a}^{2}\right)-i \gamma_{\perp}\left(3 \gamma_{\perp}^{2}+4 w_{a}^{2}-\kappa\left(2 \gamma_{\perp}^{2}-\kappa\right)\right)\right)}{\left(\left(\kappa-\gamma_{\perp}\right)^{2}+4 w_{a}^{2}\right)^{2}}, \\
& C_{4}=\frac{4 k g\left(-\left(\kappa-\gamma_{\perp}\right)+2 i w_{a}\right)}{\hbar w_{a} \gamma_{\|}\left(\left(\kappa-\gamma_{\perp}\right)^{2}+4 w_{a}^{2}\right)}, \\
& C_{5}=\frac{2 g\left(\gamma_{\|}\left(2 w_{a}^{2}+\kappa\left(\kappa-\gamma_{\|}^{2}\right)\right)-2 w_{a}^{2}\left(\kappa+\gamma_{\perp}\right)-i w_{a}\left(\gamma_{\|}\left(\kappa+\gamma_{\perp}\right)+2 \kappa\left(\kappa-\gamma_{\perp}\right)+4 w_{a}^{2}\right)\right)}{\hbar w_{a}\left(\gamma_{\|}^{2}+4 w_{a}^{2}\right)\left(\left(\kappa-\gamma_{\perp}\right)^{2}+4 w_{a}^{2}\right)}
\end{aligned}
$$

429

$$
\begin{aligned}
\frac{\partial \mathbf{A}}{\partial T} & =z_{1} \mathbf{A}+z_{2}\left(\frac{\partial}{\partial Z}+\frac{i}{2 k_{c}} \nabla_{\perp}^{2}\right) \mathbf{A}+z_{3}\left(\frac{\partial}{\partial Z}+\frac{i}{2 k_{c}} \nabla_{\perp}^{2}\right)^{2} \mathbf{A}+z_{4}\left(\mathbf{A} \cdot \mathbf{A}^{*}\right) \mathbf{A}+z_{5}(\mathbf{A} \cdot \mathbf{A}) \mathbf{A}^{*} \\
& +z_{6}\left(\mathbf{A}^{2} \cdot \mathbf{A}^{* 2}\right) \mathbf{A}+z_{7}\left(\mathbf{A}^{3} \cdot \mathbf{A}^{*}\right) \mathbf{A}^{*}
\end{aligned}
$$

$D_{2}$ is again obtained by solving Eq.(A.35):

$D_{2}=D_{20}+D_{22} e^{2 i\left(w_{a} t-k_{c} z\right)}+D_{22}^{*} e^{-2 i\left(w_{a} t-k_{c} z\right)}+D_{24} e^{4 i\left(w_{a} t-k_{c} z\right)}+D_{24}^{*} e^{-4 i\left(w_{a} t-k_{c} z\right)}$,

with

$$
\begin{aligned}
D_{20} & =\frac{4}{\hbar \mu_{0} c^{2} w_{a} \gamma_{\|}}\left(-k \mathbf{A} \mathbf{A}^{*}+\frac{k g \mathbf{A}^{2} \mathbf{A}^{* 2}}{\hbar w_{a} \gamma_{\perp}}\left(\frac{4}{\gamma_{\|}}+\frac{1}{\left(\gamma_{\|}-2 i w_{a}\right)}+\frac{1}{\left(\gamma_{\|}+2 i w_{a}\right)}\right)\right. \\
& \left.+\frac{i g \mathbf{A}^{2} \mathbf{A}^{* 2}}{\hbar w_{a} \gamma_{\perp}}\left(\frac{1}{\left(\gamma_{\|}+2 i w_{a}\right)}-\frac{1}{\left(\gamma_{\|}-2 i w_{a}\right)}\right)\right) \\
D_{22} & =\frac{2}{\hbar \mu_{0} c^{2} w_{a}\left(\gamma_{\|}+2 i w_{a}\right)}\left(-\mathbf{A}^{2}\left(k+i w_{a}\right)+\frac{2 g \mathbf{A}^{3} \mathbf{A}^{*}}{\hbar}\left(\frac{k}{\gamma_{\perp} w_{a}}\left(\frac{1}{\left(\gamma_{\|}+2 i w_{a}\right)}+\frac{2}{\gamma_{\|}}\right)\right.\right. \\
& \left.+\frac{3}{\left(\gamma_{\|}+2 i w_{a}\right)\left(8 w_{a}-3 i \gamma_{\perp}\right)}+\frac{i}{\left(\gamma_{\|}+2 i w_{a}\right)}\left(\frac{1}{\gamma_{\perp}}-\frac{3 k}{w_{a}\left(8 w_{a}-3 i \gamma_{\perp}\right)}\right)\right) \\
D_{24} & =\frac{12 g \mathbf{A}^{4}}{\hbar^{2} \mu_{0} c^{2} w_{a}\left(\gamma_{\|}+4 i w_{a}\right)\left(8 w_{a}-3 i \gamma_{\perp}\right)}\left(1-\frac{i k}{w_{a}}\right)
\end{aligned}
$$


435

436

with

$$
\begin{aligned}
z_{1}= & \frac{\mu_{0} c^{2} g \tilde{D}_{0}\left(k-\gamma_{\perp}+2 i w_{a}\right)}{\left(k-\gamma_{\perp}\right)^{2}+4 w_{a}^{2}}, \\
z_{2}= & \frac{2 c\left(2 w_{a}^{2}+\gamma_{\perp}\left(\gamma_{\perp}-k\right)+i w_{a}\left(k-3 \gamma_{\perp}\right)\right)}{\left(k-\gamma_{\perp}\right)^{2}+4 w_{a}^{2}}, \\
z_{3}= & \frac{4 c^{2} \gamma_{\perp}\left(\gamma_{\perp}^{2}\left(2 k-\gamma_{\perp}\right)+k\left(k \gamma_{\perp}-4 w_{a}^{2}\right)-i \gamma_{\perp}\left(3 \gamma_{\perp}^{2}+4 w_{a}^{2}-k\left(2 \gamma_{\perp}-k\right)\right)\right)}{\left(\left(k-\gamma_{\perp}\right)^{2}+4 w_{a}^{2}\right)^{2}}, \\
z_{4}= & \frac{4 k g\left(\left(k-\gamma_{\perp}\right)-2 i w_{a}\right)}{\hbar w_{a} \gamma_{\|}\left(\left(k-\gamma_{\perp}\right)^{2}+4 w_{a}^{2}\right)}, \\
z_{5}= & \frac{2 g\left(\gamma_{\|}\left(2 w_{a}^{2}+k\left(k-\gamma_{\perp}^{2}\right)\right)-2 w_{a}^{2}\left(k+\gamma_{\perp}\right)-i w_{a}\left(\gamma_{\|}\left(k+\gamma_{\perp}\right)+2 k\left(k-\gamma_{\perp}\right)+4 w_{a}^{2}\right)\right)}{\hbar w_{a}\left(\left(k-\gamma_{\perp}\right)^{2}+4 w_{a}^{2}\right)\left(\gamma_{\|}^{2}+4 w_{a}^{2}\right)} \\
z_{6}= & \frac{8 k g^{2}\left(\gamma_{\|}\left(k+2 \gamma_{\|}\right)+10 w_{a}^{2}\right)\left(k-\gamma_{\perp}-2 i w_{a}\right)}{\hbar^{2} w_{a}^{2} \gamma_{\perp} \gamma_{\|}^{2}\left(\left(k-\gamma_{\perp}\right)^{2}+4 w_{a}^{2}\right)\left(\gamma_{\|}^{2}+4 w_{a}^{2}\right)},
\end{aligned}
$$

$z_{7 r}=\frac{4 g^{2}\left(\begin{array}{l}\left(\gamma_{\|}\left(k\left(3 \gamma_{\|}^{2}+4 w_{a}^{2}\right)+4 w_{a}^{2} \gamma_{\|}\right)\left(9 \gamma_{\perp}^{2}+64 w_{a}^{2}\right)+3 \gamma_{\perp} \gamma_{\|}\left(\left(8 w_{a}^{2}+3 k \gamma_{\perp}\right)\left(\gamma_{\|}^{2}-4 w_{a}^{2}\right)\right.\right. \\ \left.\left.+4 \gamma_{\|} w_{a}^{2}\left(-8 k+3 \gamma_{\perp}\right)\right)\right)\left(k-\gamma_{\perp}\right)+2 w_{a}^{2}\left(\left(\left(\gamma_{\|}^{2}-4 w_{a}^{2}-4 k \gamma_{\|}\right) \gamma_{\|}-4 k\left(\gamma_{\|}^{2}+4 w_{a}^{2}\right)\right)\left(9 \gamma_{\perp}^{2}+64 w_{a}^{2}\right)\right. \\ \left.+3 \gamma_{\|} \gamma_{\perp}\left(\left(-8 k+3 \gamma_{\perp}\right)\left(\gamma_{\|}^{2}-4 w_{a}^{2}\right)-4\left(8 w_{a}^{2}+3 k \gamma_{\perp}\right) \gamma_{\|}\right)\right)\end{array}\right)}{\hbar^{2} w_{a}^{2} \gamma_{\perp} \gamma_{\|}\left(\gamma_{\|}^{2}+4 w_{a}^{2}\right)^{2}\left(9 \gamma_{\perp}^{2}+64 w_{a}^{2}\right)\left(\left(k-\gamma_{\perp}\right)^{2}+4 w_{a}^{2}\right)}$

$z_{7 i}=\frac{4 g^{2}\left(\begin{array}{l}\left(w_{a}\left(\left(\left(\gamma_{\|}^{2}-4\left(w_{a}^{2}+k \gamma_{\|}\right)\right)-4 k\left(\gamma_{\|}^{2}+4 w_{a}^{2}\right)\right)\left(9 \gamma_{\perp}^{2}+64 w_{a}^{2}\right)+3 \gamma_{\|} \gamma_{\perp}\left(\left(-8 k+3 \gamma_{\perp}\right)\left(\gamma_{\|}^{2}-4 w_{a}^{2}\right)\right.\right.\right. \\ \left.\left.-4 \gamma_{\|}\left(8 w_{a}^{2}+3 k \gamma_{\perp}\right)\right)\right)\left(\mathrm{k}-\gamma_{\perp}\right)-2\left(\gamma_{\|}\left(\mathrm{k}\left(3 \gamma_{\|}^{2}+4 w_{a}^{2}\right)+4 w_{a}^{2} \gamma_{\|}\right)\left(9 \gamma_{\perp}^{2}+64 w_{a}^{2}\right)+3 w_{a} \gamma_{\|} \gamma_{\perp}\left(\left(8 w_{a}^{2}\right.\right.\right. \\ \left.\left.\left.+3 k \gamma_{\perp}\right)\left(\gamma_{\|}^{2}-4 w_{a}^{2}\right)+4 w_{a}^{2} \gamma_{\|}\left(3 \gamma_{\perp}-8 k\right)\right)\right)\end{array}\right)}{\hbar^{2} w_{a}^{2} \gamma_{\perp} \gamma_{\|}\left(\gamma_{\|}^{2}+4 w_{a}^{2}\right)^{2}\left(9 \gamma_{\perp}^{2}+64 w_{a}^{2}\right)\left(\left(k-\gamma_{\perp}\right)^{2}+4 w_{a}^{2}\right)}$ 


\section{${ }_{437}$ References}

438

[1] F. T. Arechi, S. Boccaletti and P. L. Ramazza, Physics Reports 318, 1 (1999).

[2] J. R. Tredicce, F. T. Arecchi, G. L. Lippi, and G. P. Puccioni, J. Opt. Soc. Am. B 2, 173 (1985).

[3] S. Ciuchi, F. de Pasquale, M. San Miguel, and N.B. Abraham, Phys. Rev. A 44, 7657 (1991).

[4] E. Hernandez-Garcia, R. Toral, and M. S. Miguel, Phys. Rev. A 42, 6823 (1990).

[5] G. P. Agrawal and N. K. Dutta, Long-Wavelength Semiconductor Lasers (Van Nostrand Reinhold, New York, 1986).

[6] C. O. Weiss and R. Vilaseca, Dynamics of Lasers (VCH Publishers, Weinheim, 1991).

[7] F. Strumia, in: Advances in laser spectroscopy, eds. F.T. Arecchi, F. Strumia and H. Walther (Plenum Press, New York, 1983) p. 267.

[8] P. Colet and R. Roy, J. Opt. Lett. 19, 2056 (1994).

[9] L. Lugiato, F. Prati, and M. Brambilla, Nonlinear Optical Systems (Cambridge University Press, Cambridge, U.K., 2015).

[10] W. E. Lamb, Jr., Phys. Rev. 134, A1429 (1964).

[11] H. Haken, Laser Theory (Springer-Verlag, Berlin, 1984).

[12] I. Leyva and J. M. Guerra, Phys. Rev. A 66, 023820 (2002).

[13] F. Encinas-Sanz, I. Leyva, and J. M. Guerra, Phys. Rev. Lett. 84, 883 (2000).

[14] M. Riley, T. D. Padrick, and R. Palmer, IEEE J. Quantum Electr. QE 15, 178 (1979).

[15] P. Coullet, L. Gil and F. Rocca, Opt. Commun. 73, 403 (1989).

[16] K. Staliunas and C.O. Weiss, Physica D 81, 79 (1995). 
[17] K. Staliunas, Phys. Rev. A 48, 1573 (1993).

[18] G. L. Oppo, G. D’Alessandro and W. J. Firth, Phys. Rev. A 44, 4712 (1991).

[19] J. M. Soto-Crespo, N. N. Akhmediev, and V. V. Afanasjev, J. Opt. Soc. Am. B 13, 1439 (1996).

[20] J. Lega, J.V. Moloney and A.C. Newell, Phys. Rev. Lett. 73, 2978 (1994).

[21] M. Tlidi, M. Giorgiou, and P. Mandel, Phys. Rev. A 48, 4605 (1993).

[22] H. A. Haus and A. Mecozzi, IEEE J. Quantum Electron. 29, 983 (1993).

[23] C. R. Menyuk, J. K. Wahlstrand, J. Willits, R. P. Smith, T. R. Schibli, and S. T. Cundiff, Opt. Express 15, 6677 (2007).

[24] W.-W. Hsiang, C.-Y. Lin, and Y. Lai, Opt. Lett. 31, 1627 (2006).

[25] W. Chang, N. Akhmediev, and S. Wabnitz, Phys. Rev. A 80, 013815 (2009).

[26] J. N. Kutz, SIAM Rev. 48, 629 (2006).

[27] J. M. Soto-Crespo, N. Akhmediev, and A. Ankiewicz, Phys. Rev. Lett. 85, 2937 (2000).

[28] L. Gil, Phys. Rev. Lett. 70, 162 (1993).

[29] A. Amengual, E. Hernández-Garcia, R. Montagne and M. San Miguel, Phys. Rev. Lett. 78, 4379 (1997).

[30] E. Hernández-Garcia, M. Hoyuelos, P. Colet, M. San Miguel and R. Montagne, Int. J. Bif. Chaos 9, 2257 (1999).

[31] M. Hoyuelos, E. Hernández-Garcia, P. Colet and M. S. Miguel, Comp. Phys. Comm. 121, 414 (1999).

[32] E. Hernández-Garcia, M. Hoyuelos, P. Colet and M. S. Miguel, Phys. Rev. Lett. 85, 744 $(2000)$.

[33] M. Hoyuelos, E. Hernández-Garcia, P. Colet and M. S. Miguel, Physica D 174, 176 (2003). 
${ }_{482}$ [34] A. J. Kenfack and T. C. Kofane, J. Phys. Soc. Jpn. 72, 1800 (2003).

483

484

[35] A. Mohamadou, A. J. Kenfack, and T. C. Kofane, Phys. Rev. E 72, 036220 (2005).

[36] A. Mohamadou, B. E. Ayissi, and T. C. Kofane, Phys. Rev. E 74, 046604 (2006).

[37] C. B. Tabi, I. Maïna, A. Mohamadou, H. P. F. Ekobena and T. C. Kofané, Physica A 435, $1(2015)$.

[38] C. G. Latchio Tiofack, A. Mohamadou, T. C. Kofane, and A. B. Moubissi, Phys. Rev. E 80, 066604 (2009).

[39] G. Zakeri and E. Yomba, Phys. Rev. E 91, 062904 (2015).

[40] A. E. Siegman: Lasers (University Science Books, Mill Valley, 1986), P. 943, Eq. (50) and P. 946, Eq. (56)

[41] L. A. Lugiato, G. L. Oppo, J. R. Tredicce, L. M. Narducci, and M. A. Pernigo, J. Opt. Soc. Am. B 7, 1019 (1990)

[42] M. Hoyuelos, E. Hernández-García , P. Colet and M. S. Miguel, Physica D 174, 176 (2003)

[43] Y. Kuramoto: in Chemical Oscillations, Waves and Turbulence, ed. by H. Haken, Springer Series in Synergetics Vol. 19 (Springer, Berlin, (1984))

[44] P. H. Tatsing, A. Mohamadou, C. Bouri, C. G. Latchio Tiofack and T. C. Kofané, J. Opt. Soc. Am. B 29, 12 (2012)

[45] J. Ohtsubo: Semiconductor lasers stability, instability and chaos ed. by Springer series in optical sciences vol. 111 (Springer Verlag, (2008)) 